\title{
BOUNDS FOR THE COMPUTATIONAL POWER AND LEARNING COMPLEXITY OF ANALOG NEURAL NETS*
}

\author{
WOLFGANG MAASS ${ }^{\dagger}$
}

\begin{abstract}
It is shown that high-order feedforward neural nets of constant depth with piecewisepolynomial activation functions and arbitrary real weights can be simulated for Boolean inputs and outputs by neural nets of a somewhat larger size and depth with Heaviside gates and weights from $\{-1,0,1\}$. This provides the first known upper bound for the computational power of the former type of neural nets. It is also shown that in the case of first-order nets with piecewise-linear activation functions one can replace arbitrary real weights by rational numbers with polynomially many bits without changing the Boolean function that is computed by the neural net. In order to prove these results, we introduce two new methods for reducing nonlinear problems about weights in multilayer neural nets to linear problems for a transformed set of parameters. These transformed parameters can be interpreted as weights in a somewhat larger neural net.

As another application of our new proof technique we show that neural nets with piecewisepolynomial activation functions and a constant number of analog inputs are probably approximately correct (PAC) learnable (in Valiant's model for PAC learning [Comm. Assoc. Comput. Mach., 27 (1984), pp. 1134-1142]).
\end{abstract}

Key words. neural networks, analog computing, threshold circuits, circuit complexity, learning complexity

AMS subject classifications. 68Q05, 68Q15, 68T05, 92B20, 94C05

PII. S0097539793256041

1. Introduction. We examine in this paper the computational power and learning complexity of high-order analog feedforward neural nets $\mathcal{N}$, i.e., of circuits with analog computational elements in which certain parameters are treated as programmable parameters. We focus on neural nets $\mathcal{N}$ of bounded depth in which each gate $g$ computes a function from $\mathbf{R}^{m}$ into $\mathbf{R}$ of the form $\left\langle y_{1}, \ldots, y_{m}\right\rangle \mapsto$ $\gamma^{g}\left(Q^{g}\left(y_{1}, \ldots, y_{m}\right)\right)$. We assume that, for each gate $g, \gamma^{g}$ is some fixed piecewisepolynomial activation function (also called response function). This function is applied to some polynomial $Q^{g}\left(y_{1}, \ldots, y_{m}\right)$ of bounded degree with arbitrary real coefficients, where $y_{1}, \ldots, y_{m}$ are the real-valued inputs to gate $g$. One usually refers to the degree of the polynomial $Q^{g}$ as the order of the gate $g$. It should be noted that (following the conventions in the neural net literature) the order of a gate $g$ does not refer to the degree of its activation function $\gamma^{g}$. We will specify bounds for that degree separately.

The coefficients ("weights") of $Q^{g}$ are the programmable variables of $\mathcal{N}$ whose values may arise from some learning process.

We are primarily interested in the case where the neural net $\mathcal{N}$ computes (respectively, learns) a Boolean-valued function. For that purpose we assume that the real-valued output of the output gate $g_{\text {out }}$ of $\mathcal{N}$ is "rounded off." More precisely, we assume that there is an outer threshold $T_{\text {out }}$ (which belongs to the programmable parameters of $\mathcal{N}$ ) such that the output of $\mathcal{N}$ is 1 whenever the real-valued output $z$ of $g_{\text {out }}$ satisfies $z \geq T_{\text {out }}$ and 0 if $z<T_{\text {out }}$. In some results of this paper we also assume that the input $\left\langle x_{1}, \ldots, x_{n}\right\rangle$ of $\mathcal{N}$ is Boolean valued. It should be noted that this does

\footnotetext{
*Received by the editors September 22, 1993; accepted for publication (in revised form) July 12, 1995.

http://www.siam.org/journals/sicomp/26-3/25604.html

${ }^{\dagger}$ Institute for Theoretical Computer Science, Technische Universität Graz, Klosterwiesgasse 32/2, A-8010 Graz, Austria (maass@igi.tu-graz.ac.at).
} 
not affect the capacity of $\mathcal{N}$ to carry out, on its intermediate levels (i.e., in its "hidden units"), computation over reals, whose real-valued results are then transmitted to the next layer of gates.

Circuits of this type have rarely been considered in computational complexity theory, and they give rise to the principal question whether these intermediate analog computational elements will allow the circuit to compute more complex Boolean functions than a circuit with a similar layout but digital computational elements. Note that circuits with analog computational elements have an extra source of potentially unlimited parallelism at their disposal, since they can execute operations on numbers of arbitrary bit-length in one step, and they can transmit numbers of arbitrary bit-length from one gate to the next.

One already knows quite a bit about the special case of such neural nets $\mathcal{N}$, where each gate $g$ is a linear threshold gate. In this case each polynomial $Q^{g}\left(y_{1}, \ldots, y_{m}\right)$ is of degree $\leq 1$ (i.e., a weighted sum), and each activation function $\gamma^{g}$ in $\mathcal{N}$ is the Heaviside function (also called hard limiter) $\mathcal{H}$ defined by

$$
\mathcal{H}(y)= \begin{cases}1 & \text { if } y \geq 0 \\ 0 & \text { if } y<0\end{cases}
$$

(e.g., see $[\mathrm{R}],[\mathrm{Ni}],[\mathrm{Mu}],[\mathrm{MP}],[\mathrm{PS}],[\mathrm{HMPST}],[\mathrm{GHR}],[\mathrm{SR}],[\mathrm{SBKH}],[\mathrm{BH}],[\mathrm{A}],[\mathrm{B}]$, $[\mathrm{L}])$. The analog versus digital issue does not arise in this case, since the output of each gate is a single bit. Still, it requires some work to bound the potential power of arbitrary weights (in the weighted sums) for the computation of Boolean functions on such circuit. Since there are only finitely many Boolean circuit inputs, it is obvious that only rational weights have to be considered. The key result for the analysis of these circuits was the discovery of Muroga $[\mathrm{Mu}]$ that it is sufficient to consider for a linear threshold gate with $m$ Boolean inputs only weights $\alpha_{1}, \ldots, \alpha_{m}$ and a bias $\alpha_{0}$ that are integers of size $2^{O(m \log m)}$. (This upper bound is optimal according to a recent result of Håstad [Has].) With the help of this a priori bound on the relevant bit-length of weights, it is easy to show that the same arrays $\left(F_{n}\right)_{n \in \mathbf{N}}$ of Boolean functions $F_{n}:\{0,1\}^{n} \rightarrow\{0,1\}$ are computable by arrays $\left(\mathcal{N}_{n}\right)_{n \in \mathbf{N}}$ of neural nets of depth $O(1)$ and size $O\left(n^{O(1)}\right)$ with linear threshold gates, no matter whether one uses as weights arbitrary reals, rationals, integers, or elements of $\{-1,0,1\}$; see $[\mathrm{Mu}]$, [CSV], [HMPST], [GHR], [MT]. The resulting class of arrays $\left(F_{n}\right)_{n \in \mathbf{N}}$ of Boolean functions is called (nonuniform) $T C^{0}$ (see [HMPST], [J]).

In comparison, very little is known about upper bounds for the computational power and the learning complexity of feedforward neural nets whose gates $g$ employ more general types of activation functions $\gamma^{g}$. This holds in spite of the fact that "real neurons and real physical devices have continuous input-output relations" [Ho]. In the analysis of information processing in natural neural systems, one usually views the firing rate of a neuron as its current output. Such firing rates are known to change between a few and several hundred spikes per second (see Chap. 20 in $[\mathrm{MR}])$. Hence the activation function $\gamma^{g}$ of a gate $g$ that models such a neuron should have a graded response. It should also be noted that the customary learning algorithms for artificial neural nets (such as backward propagation $[\mathrm{RM}]$ ) are based on gradient descent methods which require that all gates $g$ employ smooth activation functions $\gamma^{g}$.

In addition, it has frequently been pointed out that it is both biologically plausible and computationally relevant to consider gates $g$ that pass to $\gamma^{g}$ instead of a weighted sum $\sum_{i=1}^{m} \alpha_{i} y_{i}+\alpha_{0}$ some polynomial $Q^{g}\left(y_{1}, \ldots, y_{m}\right)$ of bounded degree, where $y_{1}, \ldots, y_{m}$ are circuit inputs or outputs of the immediate predecessors 
of $g$. Such gates are called sigma-pi units or high-order gates in the literature (see p. 73 and Chap. 10 in $[\mathrm{RM}]$; see also $[\mathrm{DR}],[\mathrm{H}],[\mathrm{PG}],[\mathrm{MD}])$. From the point of view of approximation theory there has been particular interest in the case in which $Q^{g}\left(y_{1}, \ldots, y_{m}\right)=\sum_{i=1}^{m} \alpha_{i}\left(y_{i}-c_{i}\right)^{2}$ measures a "distance" of its input $\left\langle y_{1}, \ldots, y_{m}\right\rangle$ from some "center" $\left\langle c_{1}, \ldots, c_{m}\right\rangle$ (the latter may be determined through a learning process). Apparently Theorems 3.1 and 4.3 of this paper provide the first upper bounds for the computational power and learning complexity of high-order feedforward neural nets with non-Boolean activation functions.

The power of feedforward neural nets with other activation functions besides $\mathcal{H}$ has previously been investigated in [RM, Chap. 10], [S1], [S2], [H], [MSS], [DS], [SS]. It was shown in [MSS] for a very general class of activation functions $\gamma^{g}$ that neural nets $\left(\mathcal{N}_{n}\right)_{n \in \mathbf{N}}$ of constant depth and size $O\left(n^{O(1)}\right)$ with real weights of size $O\left(n^{O(1)}\right)$ and output separation $\Omega\left(1 / n^{O(1)}\right)$ (between the unrounded circuit outputs for rejected and accepted inputs) can compute only Boolean functions in $T C^{0}$. It follows from a result of Sontag [S2] that the assumptions on the weight size and separation are essential for this upper bound: he constructed an arbitrarily smooth monotone function $\Theta$ (which can be made to satisfy the conditions on $\gamma^{g}$ in the quoted result of [MSS]) and neural nets $\mathcal{N}_{n}$ of size 2 (!) with activation function $\Theta$ such that $\mathcal{N}_{n}$ can compute with sufficiently large weights any Boolean function $F_{n}:\{0,1\}^{n} \rightarrow\{0,1\}$ (hence $\mathcal{N}_{n}$ has $\mathrm{VC}$ dimension $2^{n}$ ).

These results leave open the question about the computational power and learning complexity of feedforward neural nets with arbitrary weights that employ "natural" analog activation functions $\gamma^{g}$. For example there has previously been no upper bound for the set of Boolean functions computable by analog neural nets with the very simple piecewise-linear activation function $\pi$ defined by

$$
\pi(y)= \begin{cases}0 & \text { if } y \leq 0 \\ y & \text { if } 0 \leq y \leq 1 \\ 1 & \text { if } y \geq 1\end{cases}
$$

([L] refers to a gate $g$ with $\gamma^{g}=\pi$ as a threshold logic element.) On the other hand, there exist results which suggest that such upper bound would be nontrivial. It has already been shown in [MSS] that constant size neural nets of depth 2 with activation function $\pi$ and small integer weights can compute more Boolean functions than constant size neural nets of depth 2 with linear threshold gates (and arbitrary weights). [DS] exhibits an even stronger increase in computational power for the case of quadratic activation functions.

Hence even simple non-Boolean activation functions provide more computational power to a neural net than the Heaviside function. However it has been an open problem by how much they can increase the computational power (in the presence of arbitrary weights). From the technical point of view, this difficulty in proving an upper bound for the computational power was caused by the lack of an upper bound on the amount of information that can be encoded in such a neural net by the assignment of weights. For the case of neural nets with Heaviside gates, this upper bound on the information capacity of weights is provided by the quoted result of Muroga $[\mathrm{Mu}]$. However, this problem is substantially more difficult for neural nets with piecewise-linear activation functions. For this model it is no longer sufficient to analyze a single gate with Boolean inputs and outputs. Even if the inputs and outputs of the neural net are Boolean valued, the signals that are transmitted between the hidden units are real valued. Furthermore, one can give no a priori bound on the 
precision required for such analog signals between hidden units, since one has no control over the maximal size of weights in the neural net. Obviously a large weight will magnify any imprecision. Note also that a computation on a multilayer neural net of the type considered here involves products of weights from subsequent levels. Hence, if some of the weights are arbitrarily large, one needs arbitrarily high precision for the other weights.

The main technical contribution of this paper is two new methods for reducing nonlinear problems about weights in multilayer neural nets to linear problems for a transformed set of parameters. These two methods are presented in sections 2 and 3 of this paper. We introduce in section 2 of this paper a method that allows us to prove an upper bound for the information capacity of weights for neural nets with piecewise-linear activation functions (hence in particular for $\pi$ ). It is shown that for the computation of Boolean functions on neural nets $\mathcal{N}_{n}$ of constant depth and polynomially in $n$ many gates (where $n$ is the number of input variables) it is sufficient to use as weights rational numbers with polynomially in $n$ many bits. As a consequence, one can simulate any such analog neural net by a digital neural net of constant depth and polynomial size with the Heaviside activation function (i.e., linear threshold gates) and binary weights (i.e., weights from $\{0,1\}$ ). This result also implies that the VC dimension of $\mathcal{N}_{n}$ can be bounded above by a polynomial in $n$.

In section 3 we introduce another proof technique that allows us to derive the same two consequences for neural nets with piecewise-polynomial activation functions and nonlinear gate inputs $Q^{g}\left(y_{1}, \ldots, y_{m}\right)$ of bounded degree. These results show that in spite of the previously quoted evidence for the superiority of non-Boolean activation functions in neural nets, there is some limit to their computational power as long as the activation functions are piecewise-polynomial. On the other hand the polynomial upper bound on the VC dimension of such neural nets may be interpreted as good news: it shows that neural nets of this type can in principle be trained with a sequence of examples that is not too long.

We conclude in section 4 with a positive result for learning on neural nets in Valiant's model [V] for probably approximately correct learning (PAC learning). We consider the problem of learning on neural nets with a fixed number of analog (i.e., real-valued) input variables. We exploit here the implicit linearization of the requirements for the desired weight assignment that is achieved in the new proof techniques from sections 2 and 3 . In this way one can show that such neural nets are properly PAC learnable in the case of piecewise-linear activation functions and PAC learnable with a hypothesis class that is given by a somewhat larger neural net in the case of piecewise-polynomial activation functions. Another application of our parameter transformation method from section 2 to PAC learning has subsequently been given by Koiran [K94].

The results of this paper were first announced in [M92], and an extended abstract of these results appeared in [M93a]. Another result of [M93a], the construction of neural nets whose VC dimension is superlinear in the number of weights, has subsequently been improved to apply for depth 3 also. A full version of that proof appears in [M93b].

Definition 1.1. A network architecture (or neural net) $\mathcal{N}$ of order $k$ is a labeled acyclic directed graph $\langle V, E\rangle$. Its nodes of fan-in 0 are labeled by the input variables $x_{1}, \ldots, x_{n}$. Each node $g$ of fan-in $m>0$ is called a computation node (or gate) and is labeled by some activation function $\gamma^{g}: \boldsymbol{R} \rightarrow \boldsymbol{R}$ and some polynomial $Q^{g}\left(y_{1}, \ldots, y_{m}\right)$ of degree $\leq k$. Furthermore, $\mathcal{N}$ has a unique node of fan-out 0 which is called the 
output node of $\mathcal{N}$ and which carries as an additional label a certain real number $T_{\text {out }}$ (called the outer threshold of $\mathcal{N}$ ).

The coefficients of all polynomials $Q^{g}\left(y_{1}, \ldots, y_{m}\right)$ for gates $g$ in $\mathcal{N}$ and the outer threshold $T_{\text {out }}$ are called the programmable parameters of $\mathcal{N}$. Assume that $\mathcal{N}$ has $w$ programmable parameters and that some numbering of these has been fixed. Then each assignment $\alpha \in \boldsymbol{R}^{w}$ of reals to the programmable parameters in $\mathcal{N}$ defines an analog circuit $\mathcal{N}^{\alpha}$, which computes a function $x \mapsto \mathcal{N}^{\alpha}(x)$ from $\boldsymbol{R}^{n}$ into $\{0,1\}$ in the following way: assume that some input $x \in \boldsymbol{R}^{n}$ has been assigned to the input nodes of $\mathcal{N}$. If a gate $g$ in $\mathcal{N}$ has $m$ immediate predecessors in $\langle V, E\rangle$ which output $y_{1}, \ldots, y_{m} \in \boldsymbol{R}$, then $g$ outputs $\gamma^{g}\left(Q^{g}\left(y_{1}, \ldots, y_{m}\right)\right)$. Finally, if $g_{\text {out }}$ is the output gate of $\mathcal{N}$ and $g_{\text {out }}$ gives the real-valued output $z$ (according to the preceding inductive definition), we define

$$
\mathcal{N}^{\alpha}(x):= \begin{cases}1 & \text { if } z \geq T_{\text {out }} \\ 0 & \text { if } z<T_{\text {out }}\end{cases}
$$

where $T_{\text {out }}$ is the outer threshold that has been assigned by $\alpha$ to $g_{\text {out }}$.

Any parameters that occur in the definitions of the activation functions $\gamma^{g}$ of $\mathcal{N}$ are referred to as architectural parameters of $\mathcal{N}$.

Definition 1.2. A function $\gamma: \boldsymbol{R} \rightarrow \boldsymbol{R}$ is called piecewise-polynomial if there are thresholds $t_{1}, \ldots, t_{k} \in \boldsymbol{R}$ and polynomials $P_{0}, \ldots, P_{k}$ such that $t_{1}<\cdots<t_{k}$ and for each $i \in\{0, \ldots, k\}: t_{i} \leq x<t_{i+1} \Rightarrow \gamma(x)=P_{i}(x)$ (we set $t_{0}:=-\infty$ and $\left.t_{k+1}:=\infty\right)$.

If $k$ is chosen minimal for $\gamma$, we refer to $k$ as the number of polynomial pieces of $\gamma$; to $P_{0}, \ldots, P_{k}$ as the polynomial pieces of $\gamma$; and to $t_{1}, \ldots, t_{k}$ as the thresholds of $\gamma$. Furthermore we refer to $t_{1}, \ldots, t_{k}$ together with all coefficients in the polynomials $P_{0}, \ldots, P_{k}$ as the parameters of $\gamma$. The maximal degree of $P_{0}, \ldots, P_{k}$ is called the degree of $\gamma$. If the degree of $\gamma$ is $\leq 1$ then we call $\gamma$ piecewise-linear, and we refer to $P_{0}, \ldots, P_{k}$ as the linear pieces of $\gamma$.

If $\gamma$ occurs as activation function $\gamma^{g}$ of some network architecture $\mathcal{N}$, then one refers to the parameters of $\gamma$ as architectural parameters of $\mathcal{N}$.

Note that we do not require that $\gamma$ is continuous (or monotone). It should also be pointed out that according to Definition 1.1 the order $k$ of a neural net does not bound the degrees of the polynomial pieces of its activation functions. Finally, we would like to mention that in contrast to [MSS], we do not require here any minimal distance between the real-valued network outputs $z$ and the outer threshold $T_{\text {out }}$.

DEFINITION 1.3. Assume that $\mathcal{N}$ is an arbitrary network architecture with $n$ inputs and $w$ programmable parameters and that $S \subseteq \boldsymbol{R}^{n}$ is an arbitrary set. Then one defines the $\mathrm{VC}$ dimension of $\mathcal{N}$ over $S$ in the following way:

$$
\begin{aligned}
V C \text { dimension }(\mathcal{N}, S):= & \max \left\{\left|S^{\prime}\right| \mid S^{\prime} \subseteq S\right. \text { has the property that for every function } \\
& F: S^{\prime} \rightarrow\{0,1\} \text { there exists a parameter assignment } \\
& \left.\alpha \in \boldsymbol{R}^{w} \text { such that } \forall x \in S^{\prime}\left(\mathcal{N}^{\alpha}(x)=F(x)\right)\right\} .
\end{aligned}
$$

Remark 1.4. VC dimension is an abbreviation for Vapnik-Chervonenkis dimension. It has been shown in $[\mathrm{BEHW}]$ (see also $[\mathrm{BH}],[\mathrm{A}]$ ) that the $\mathrm{VC}$ dimension of a neural net $\mathcal{N}$ essentially determines the number of examples that are needed to train $\mathcal{N}$ (in Valiant's model for PAC learning [V]). Sontag [S2] has shown that the VC dimension of a neural net can be drastically increased by using activation functions with non-Boolean output instead of the Heaviside function $\mathcal{H}$. The methods described in 
this paper were used in [M93a] to give the first proof of a polynomial upper bound for the $\mathrm{VC}$ dimension of the here-considered neural nets. Subsequently [GJ] have shown that such bounds for the VC dimension can also be derived more directly via Milnor's theorem. However their method does not yield upper bounds for the computational power of these neural nets.

2. A bound for the information-capacity of weights in neural nets with piecewise-linear activation functions. We consider for arbitrary $a \in \mathbf{N}$ the following set of rationals with up to $a$ bits before and after the comma:

$$
\begin{gathered}
\mathbf{Q}_{a}:=\left\{r \in \mathbf{Q} \mid \quad r=s \cdot \sum_{i=-a}^{a-1} b_{i} \cdot 2^{i} \quad \text { for } b_{i} \in\{0,1\}, i=-a, \ldots, a-1,\right. \text { and } \\
s \in\{-1,1\}\} .
\end{gathered}
$$

Note that for any $r \in \mathbf{Q}_{a}:|r| \leq 2^{a} \leq 2^{2 a} \cdot \min \left\{\left|r^{\prime}\right| \mid r^{\prime} \in \mathbf{Q}_{a}\right.$ and $\left.r^{\prime} \neq 0\right\}$.

THEOREM 2.1. Consider an arbitrary network architecture $\mathcal{N}$ of order 1 over a graph $\langle V, E\rangle$ with $n$ input nodes in which every computation node has fan-out $\leq 1$. Assume that each activation function $\gamma^{g}$ in $\mathcal{N}$ is piecewise-linear with parameters from $\boldsymbol{Q}_{a}$. Let $w:=|V|+|E|+1$ be the number of programmable parameters in $\mathcal{N}$.

Then for every $\alpha \in \boldsymbol{R}^{w}$ there exists a vector $\alpha^{\prime}=\left\langle\begin{array}{c}s_{1} \\ t\end{array}, \ldots, \frac{s_{w}}{t}\right\rangle \in \boldsymbol{Q}^{w}$ with integers $s_{1}, \ldots, s_{w}, t$ of absolute value $\leq(2 w+1) ! 2^{2 a(2 w+1)}$ such that $\forall x \in \boldsymbol{Q}_{a}^{n}\left(\mathcal{N}^{\alpha}(x)=\right.$ $\mathcal{N}^{\alpha^{\prime}}(x)$ ). In particular $\mathcal{N}^{\alpha^{\prime}}$ computes the same Boolean function as $\mathcal{N}^{\alpha}$.

Remark 2.2. The condition of Theorem 2.1 that all computation nodes in $\mathcal{N}$ have fan-out $\leq 1$ is automatically satisfied for $d \leq 2$. For larger $d$ one can simulate any network architecture $\mathcal{N}$ of depth $d$ with $s$ nodes by a network architecture $\mathcal{N}^{\prime}$ with $\leq \frac{s}{s-1} \cdot s^{d-1} \leq{ }_{2}^{3} s^{d-1}$ nodes and depth $d$ that satisfies this condition (replace each computation node with fan-out $k$ by $k$ identical nodes with fan-out 1 , starting from the output layer). Hence this condition is not too restrictive for network architectures of a constant depth $d$.

It should also be pointed out that there is in the assumption of Theorem 2.1 no explicit bound on the number of linear pieces of $\gamma^{g}$ (apart from the requirement that its thresholds are from $\mathbf{Q}_{a}$ ). For example, these activation functions may consist of $2^{a}$ linear pieces (with discontinuous jumps in between). Furthermore $\gamma^{g}$ is not required to be monotone.

Finally, it should be mentioned that a corresponding version of Theorem 2.1 also holds for rational numbers that do not have a finite binary representation, i.e., for all rationals from $\mathbf{Q}_{a}^{\prime}:=\{r \in \mathbf{Q}: r$ is the quotient of integers of bit-length $\leq a\}$ instead of $\mathbf{Q}_{a}$.

Remark 2.3. Previously, one had no upper bound for the computational power (or for the $\mathrm{VC}$ dimension) of multilayer neural nets $\mathcal{N}$ with arbitrary weights and analog computational elements (i.e., activation functions with non-Boolean output). Theorem 2.1 implies that any $\mathcal{N}$ of the considered type can compute with the help of arbitrary parameter assignments $\alpha \in \mathbf{R}^{w}$ at most $2^{O\left(a w^{2} \log w\right)}$ different functions from $\mathbf{Q}_{a}^{n}$ into $\{0,1\}$, hence $\mathrm{VC}$ dimension $\left(\mathcal{N}, \mathbf{Q}_{a}^{n}\right)=O\left(w^{2}(a+\log w)\right.$ ) (see Remark 3.9 for a slightly better bound and for a related bound for the case of inputs from $\left.\mathbf{R}^{n}\right)$.

Furthermore Theorem 2.1 implies that one can replace all analog computations inside $\mathcal{N}$ by digital arithmetical operations on not too large integers (the proof gives an upper bound of $O(w a+w \log w)$ for their bit-length). It is well known that each of 
these digital arithmetical operations (multiple addition, multiplication, division) can be carried out on a circuit of small constant depth with $O\left(a^{O(1)} \cdot w^{O(1)}\right)$ MAJORITYgates, hence also on a network architecture of depth $O(1)$ and size $O\left(a^{O(1)} \cdot w^{O(1)}\right)$ with Heaviside gates and weights from $\{-1,0,1\}$ (see [CSV], [PS], [HMPST], [GHR], $[\mathrm{SR}],[\mathrm{SBKH}])$. Thus one can simulate for inputs from $\{0,1\}^{n}$ any depth $d$ network architecture $\mathcal{N}$ as in Theorem 2.1 with arbitrary parameter assignments $\alpha \in \mathbf{R}^{w}$ by a network architecture of depth $O(d)$ and size $O\left(a^{O(1)} \cdot w^{O(1)}\right)$ with Heaviside gates and weights from $\{-1,0,1\}$. The same holds for inputs from $\mathbf{Q}_{a}^{n}$ if they are given to $\mathcal{N}$ in digital form.

The size of this simulating digital neural net with Heaviside gates is polynomial in the number of real-valued parameters of the simulated analog neural net $\mathcal{N}$ but exponential in the depth of $\mathcal{N}$. Subsequent to [M93a], Koiran [K93] has proven a complementary simulation result, where the size of the simulating digital neural net is exponential in the number of real-valued parameters in $\mathcal{N}$ but subexponential in the depth of $\mathcal{N}$.

Proof of Theorem 2.1. In the special case where $\gamma^{g}=\mathcal{H}$ for all gates in $\mathcal{N}$ this result is well known $[\mathrm{Mu}]$. It follows by applying separately to each gate in $\mathcal{N}$ the following result.

LEMMA 2.4 (folklore; see [MT] for a proof). Consider a system $A x \leq b$ of some arbitrary finite number of linear inequalities in l variables. Assume that all entries in $A$ and $b$ are integers of absolute value $\leq K$.

If this system has any solution in $\boldsymbol{R}^{\bar{l}}$, then it has a solution of the form $\left\langle s_{1}, \ldots,{ }_{t} s_{t}\right\rangle$, where $s_{1}, \ldots, s_{l}, t$ are integers of absolute value $\leq(2 l+1) ! K^{2 l+1}$.

Sketch of the proof for Lemma 2.4. Let $k$ be the number of inequalities in $A x \leq b$. One writes each variable in $x$ as a difference of two nonnegative variables and adds to each inequality a "slack variable." In this way one gets an equivalent system

$$
A^{\prime} x^{\prime}=b, \quad x^{\prime} \geq 0
$$

over $l^{\prime}:=2 l+k$ variables for some $k \times l^{\prime}$ matrix $A^{\prime}$. The $k$ columns of $A^{\prime}$ for the $k$ slack variables in $x^{\prime}$ form an identity matrix. Hence $A^{\prime}$ has rank $k$.

The assumption of the lemma implies that (1) has a solution over R. Hence by Carathéodory's theorem (Corollary $7.1 \mathrm{i}$ in $[\mathrm{Sch}]$ ) one can conclude that there is also a solution over $\mathbf{R}$ of a system

$$
A^{\prime \prime} x^{\prime \prime}=b, \quad x^{\prime \prime} \geq 0 .
$$

Subsequent to the first publication of the techniques of this article in [M93a], Koiran [K93] has proven a complementary result without assumption that the depth is bounded but where one has to assume that the number of real-valued parameters in the given neural net $\mathcal{N}$ is bounded by a constant where $A^{\prime \prime}$ consists of $k$ linearly independent columns of $A^{\prime}$. Since $A^{\prime \prime}$ has full rank, (2) has in fact a unique solution that is given by Cramer's rule: $x_{j}^{\prime \prime}=\operatorname{det}\left(A_{j}^{\prime \prime}\right) / \operatorname{det} A^{\prime \prime}$ for $j=1, \ldots, k$, where $A_{j}^{\prime \prime}$ results form $A^{\prime \prime}$ by replacing its $j$ th column by $b$. Since all except up to $2 l$ columns of $A^{\prime \prime}$ contain exactly one 1 and else only 0 's, we can bring each of the matrices $A^{\prime \prime}$, $A_{j}^{\prime \prime}$ by permutations of rows and columns into a form

$$
B=\left(\begin{array}{ll}
C & 0 \\
D & I
\end{array}\right),
$$

where $C$ is a square matrix with $2 l+1$ rows. Hence the determinant of $B$ is an integer of absolute value $\leq(2 l+1) ! K^{2 l+1}$. 
The difficulty of the proof of Theorem 2.1 lies in the fact that with analog computational elements one can no longer treat each gate separately, since intermediate values are no longer integers. Furthermore, the total computation of $\mathcal{N}$ can in general not be described by a system of linear inequalities, where the $w$ variable parameters of $\mathcal{N}$ are the variables in the inequalities (and the fixed parameters of $\mathcal{N}$ are the constants). This becomes obvious if one just considers the composition of two very simple analog gates $g_{1}$ and $g_{2}$ on levels 1 and 2 of $\mathcal{N}$, whose activation functions $\gamma_{1}, \gamma_{2}$ satisfy $\gamma_{1}(y)=\gamma_{2}(y)=y$. Assume $x=\sum_{i=1}^{n} \alpha_{i} x_{i}+\alpha_{0}$ is the input to gate $g_{1}$, and $g_{2}$ receives as input $\sum_{j=1}^{m} \alpha_{j}^{\prime} y_{j}+\alpha_{0}^{\prime}$, where $y_{1}=\gamma_{1}(x)=x$ is the output of gate $g_{1}$. Then $g_{2}$ outputs $\alpha_{1}^{\prime} \cdot\left(\sum_{i=1}^{n} \alpha_{i} x_{i}+\alpha_{0}\right)+\sum_{j=2}^{m} \alpha_{j}^{\prime} y_{j}+\alpha_{0}^{\prime}$. Obviously this term is not linear in the weights $\alpha_{1}^{\prime}, \alpha_{1}, \ldots, \alpha_{n}$. Hence if the output of gate $g_{2}$ is compared with a fixed threshold at the next gate, the resulting inequality is not linear in the weights of the gates in $\mathcal{N}$.

If the activation functions of all gates in $\mathcal{N}$ were linear (as in the example for $g_{1}$ and $g_{2}$ ), then there would be no problem because a composition of linear functions is linear. However for piecewise-linear activation functions it is not sufficient to consider their composition, since intermediate results have to be compared with boundaries between linear pieces of the next gate.

We introduce in this paper a new method in order to handle this difficulty. We simulate $\mathcal{N}^{\alpha}$ by another neural net $\hat{\mathcal{N}}[c]^{\beta}$ (which one may view as a "normal form" for $\mathcal{N}^{\alpha}$ ) that uses the same graph $\langle V, E\rangle$ as $\mathcal{N}$ but different activation functions and different values $\beta$ for its variable parameters. The activation functions of $\hat{\mathcal{N}}[c]$ depend on $|V|$ new parameters $c \in \mathbf{R}^{|V|}$, which we call scaling parameters in the following. Although this new neural net has the disadvantage that it requires $|V|$ additional parameters $c$, it has the advantage that we can choose in $\hat{\mathcal{N}}[c]$ all weights on edges between computation nodes to be from $\{-1,0,1\}$. Since these weights from $\{-1,0,1\}$ are already of the desired bit-length, we can treat them as constants in the system of inequalities that describes computations of $\hat{\mathcal{N}}[c]$. Therefore, all variables that appear in the inequalities that describe computations of $\hat{\mathcal{N}}[c]$ (the variables for weights of gates on level 1 , the variables for the biases of gates on all levels, the variable for the outer threshold, and the new variables for the scaling parameters $c$ ) appear only linearly in those inequalities. Hence we can apply Lemma 2.4 to the system of inequalities that describes the computations of $\hat{\mathcal{N}}$ for inputs from $\mathbf{Q}_{a}^{n}$ and thereby get a rational solution $\beta^{\prime}, c^{\prime}$ for all variable parameters in $\hat{\mathcal{N}}$. Finally we observe that we can transform $\hat{\mathcal{N}}\left[c^{\prime}\right]^{\beta^{\prime}}$ back into the original neural net $\mathcal{N}$ with an assignment of rational numbers $\alpha^{\prime}$ to all variable parameters in $\mathcal{N}$.

We will now fill in some of the missing details. Consider the gate function $\gamma$ of an arbitrary gate $g$ in $\mathcal{N}$. Since $\gamma$ is piecewise-linear, there are fixed parameters $t_{1}<\cdots<t_{k}, a_{0}, \ldots, a_{k}, b_{0}, \ldots, b_{k}$ in $\mathbf{Q}_{a}$ (which may be different for different gates $g$ ) such that with $t_{0}:=-\infty$ and $t_{k+1}:=+\infty$ one has $\gamma(x)=a_{i} x+b_{i}$ for $x \in \mathbf{R}$ with $t_{i} \leq x<t_{i+1} ; i=0, \ldots, k$. For an arbitrary scaling parameter $c \in \mathbf{R}^{+}$we associate with $\gamma$ the following piecewise-linear activation function $\gamma^{c}$ : the thresholds of $\gamma^{c}$ are $c \cdot t_{1}, \ldots, c \cdot t_{k}$, and its output is $\gamma^{c}(x)=a_{i} x+c \cdot b_{i}$ for $x \in \mathbf{R}$ with $c \cdot t_{i} \leq x<c \cdot t_{i+1}$; $i=0, \ldots, k$ (set $\left.c \cdot t_{0}:=-\infty, c \cdot t_{k+1}:=+\infty\right)$. Thus for all reals $c>0$ the function $\gamma^{c}$ is related to $\gamma$ through the equality: $\forall x \in \mathbf{R}\left(\gamma^{c}(c \cdot x)=c \cdot \gamma(x)\right)$.

Assume that $\alpha \in \mathbf{R}^{w}$ is some arbitrarily given assignment to the variable parameters in $\mathcal{N}$. We transform $\mathcal{N}^{\alpha}$ into a normal form $\hat{\mathcal{N}}[c]^{\beta}$, in which all weights on edges between computation nodes are from $\{-1,0,1\}$ such that $\forall x \in \mathbf{R}^{n}\left(\mathcal{N}^{\alpha}(x)=\right.$ $\left.\hat{\mathcal{N}}[c]^{\beta}(x)\right)$. We proceed inductively from the output level towards the input level. 
Assume that the output gate $g_{\text {out }}$ of $\mathcal{N}^{\alpha}$ receives as input $\sum_{i=1}^{m} \alpha_{i} y_{i}+\alpha_{0}$, where $\alpha_{1}, \ldots, \alpha_{m}, \alpha_{0}$ are the weights and the bias of $g_{\text {out }}$ (under the assignment $\alpha$ ) and $y_{1}, \ldots, y_{m}$ are the (real-valued) outputs of the immediate predecessors $g_{1}, \ldots, g_{m}$ of $g$. For each $i \in\{1, \ldots, m\}$ with $\alpha_{i} \neq 0$ such that $g_{i}$ is not an input node we replace the activation function $\gamma_{i}$ of $g_{i}$ by $\gamma_{i}^{\left|\alpha_{i}\right|}$, and we multiply the weights and the bias of gate $g_{i}$ with $\left|\alpha_{i}\right|$. Finally we replace the weight $\alpha_{i}$ of gate $g_{\text {out }}$ by

$$
\operatorname{sgn}\left(\alpha_{i}\right):=\left\{\begin{aligned}
1 & \text { if } \alpha_{i}>0 \\
-1 & \text { if } \alpha_{i}<0
\end{aligned}\right.
$$

This operation has the effect that the multiplication with $\left|\alpha_{i}\right|$ is carried out before the gate $g_{i}$ (rather than after $g_{i}$, as done in $\mathcal{N}^{\alpha}$ ) but that the considered output gate $g_{\text {out }}$ still receives the same input as before. The analogous operation is then inductively carried out for the predecessors $g_{i}$ of $g_{\text {out }}$ (note, however, that the weights of $g_{i}$ are no longer the original ones from $\mathcal{N}^{\alpha}$, since they have been changed in the preceding step). We exploit here the assumption that each gate has fan-out $\leq 1$.

Let $\beta$ consist of the new weights on edges adjacent to input nodes, the resulting biases of all gates in $\hat{\mathcal{N}}$, and the (unchanged) outer threshold $T_{\text {out }}$. Let $c$ consist of the resulting scaling factors at the gates of $\mathcal{N}$. Then we have $\forall x \in \mathbf{R}^{n}\left(\mathcal{N}^{\alpha}(x)=\right.$ $\left.\hat{\mathcal{N}}[c]^{\beta}(x)\right)$.

Finally we have to replace all strict inequalities of the form " $s_{1}<s_{2}$ " that are needed to describe the computation of $\hat{\mathcal{N}}[c]^{\beta}$ for some input $x \in \mathbf{Q}_{a}^{n}$ by inequalities of the form " $s_{1}+1 \leq s_{2}$ ". This concerns inequalities of the form $s<c \cdot t_{i}$, where $c \cdot t_{i}$ is the threshold of some gate $g$ in $\hat{\mathcal{N}}[c]$ and $s$ is its gate input, inequalities of the form $s<T_{\text {out }}$ where $s$ is the output of $g_{\text {out }}$, and inequalities of the form $0<c$ for each scaling parameter $c$. In order to achieve this stronger separation it is sufficient to multiply all parameters $\beta, c$ in $\hat{\mathcal{N}}$ by a sufficiently large constant $K$. For simplicity we write again $\beta, c$ for the resulting parameters. We now specify a system $\mathcal{A} z \leq b$ of linear inequalities in $w$ variables $z$ that play the role of the $w$ parameters $\beta, c$ in the computations of $\hat{\mathcal{N}}[c]^{\beta}$ for all inputs $x$ from $\mathbf{Q}_{a}^{n}$. The constants of these inequalities are the coordinates of all inputs $x \in \mathbf{Q}_{a}^{n}$, the parameters of the activation functions $\gamma$ in $\mathcal{N}$, the constants $-1,1$ that occur in $\hat{\mathcal{N}}$ as weights of edges between computation nodes, and the constants 1 that arise from the replacement of strict inequalities " $s_{1}<s_{2}$ " by " $s_{1}+1 \leq s_{2}$."

For each fixed input $x \in \mathbf{Q}_{a}^{n}$ one places into the system $\mathcal{A} z \leq b$ up to two linear inequalities for each gate $g$ in $\mathcal{N}$. These inequalities are defined by induction on the depth of $g$. If $g$ has depth $1, t_{1}<\cdots<t_{k}$ are the thresholds of its activation functions $\gamma$ in $\mathcal{N}$, and its input $\sum_{i=1}^{n} \alpha_{i} x_{i}+\alpha_{0}$ in $\hat{\mathcal{N}}[c]^{\beta}$ satisfies $c \cdot t_{j} \leq \sum_{i=1}^{n} \alpha_{i} x_{i}+\alpha_{0}$ and $\sum_{i=1}^{n} \alpha_{i} x_{i}+\alpha_{0}+1 \leq c \cdot t_{j+1}$, then one adds these two inequalities to the system (more precisely, if $j=0$ or $j=k$ then only one inequality is needed since the other one is automatically true).

If $g^{\prime}$ is a successor gate of $g$, it receives from $g$ for some specific $j \in\{0, \ldots, k\}$ an output of the form $a_{j} \cdot\left(\sum_{i=1}^{n} \alpha_{i} x_{i}+\alpha_{0}\right)+c \cdot b_{j}$ (where $c$ is the scaling factor of gate $g$ ). Note that this term is linear, since $a_{j}, b_{j}$ are fixed parameters of gate $g^{\prime}$. In this way one can express for circuit input $x$ the input $I(x)$ of gate $g^{\prime}$ as a linear term in the weights, biases, and scaling factors of its preceding gates. (We exploit here that in $\hat{\mathcal{N}}$ the weight on the edge between $g^{\prime}$ and each predecessor gate is a fixed parameter from $\{-1,0,1\}$, not a variable.) If this input $I(x)$ satisfies in $\hat{\mathcal{N}}[c]^{\beta}$ the inequalities $c^{\prime} \cdot t_{j^{\prime}}^{\prime} \leq I(x)$ and $I(x)+1 \leq c^{\prime} \cdot t_{j^{\prime}+1}^{\prime}$ (where $t_{1}^{\prime}<\cdots<t_{k^{\prime}}^{\prime}$ are the thresholds of $g^{\prime}$ in $\mathcal{N}$, and $c^{\prime}$ is the scaling factor of $g^{\prime}$ in $\hat{\mathcal{N}}$ ), then one adds these two 
inequalities to the system $\mathcal{A} z \leq b$ (respectively, only one if $j^{\prime}=0$ or $j^{\prime}=k^{\prime}$ ). Note that all resulting inequalities are linear, in spite of the fact that the system contains variables for the biases of all gates. It should also be pointed out that the definition of this system of inequalities is more involved than it may first appear, since the sum of terms $I(x)$ depends on the chosen inequalities for all predecessor gates (e.g., on $j$ in the example above). Hence a precise definition has to be similar to that of the proof of Theorem 3.1.

It is clear that the resulting system $\mathcal{A} z \leq b$ has a solution in $\mathbf{R}^{w}$, since $z:=\langle\beta, c\rangle$ is a solution. Hence we can apply Lemma 2.4, which provides a solution $z^{\prime}$ of the form $\left\langle{ }_{t}^{s_{i}}\right\rangle_{i=1, \ldots, w}$ with integers $s_{1}, \ldots, s_{w}, t$ of absolute value $\leq(2 w+1) ! 2^{2 a(2 w+1)}$. Let $\hat{\mathcal{N}}\left[c^{\prime}\right]^{\beta^{\prime}}$ be the neural net $\hat{\mathcal{N}}$ with this new assignment $\left\langle\beta^{\prime}, c^{\prime}\right\rangle:=z^{\prime}$ of "small" parameters. By definition we have $\forall x \in \mathbf{Q}_{a}^{n}\left(\mathcal{N}^{\alpha}(x)=\hat{\mathcal{N}}\left[c^{\prime}\right]^{\beta^{\prime}}\right)$. We show that one can transform this neural net $\hat{\mathcal{N}}\left[c^{\prime}\right]^{\beta^{\prime}}$ into a net $\mathcal{N}^{\beta^{\prime}}$ with the same activation functions as $\mathcal{N}^{\alpha}$ but a new assignment $\alpha^{\prime}$ of rational parameters (that can easily be computed from $\beta^{\prime}, c^{\prime}$ ). This transformation proceeds inductively from the input level towards the output level. Consider some gate $g$ on level 1 in $\hat{\mathcal{N}}$ that uses (for the new parameter assignment $c^{\prime}$ ) the scaling factor $c>0$ for its activation function $\gamma^{c}$. Then we replace the weights $\alpha_{1}, \ldots, \alpha_{n}$ and bias $\alpha_{0}$ of gate $g$ in $\hat{\mathcal{N}}\left[c^{\prime}\right]^{\beta^{\prime}}$ by ${ }_{c}^{\alpha_{i}}, \ldots, \frac{\alpha_{n}}{c}, \frac{\alpha_{0}}{c}$ and $\gamma^{c}$ by $\gamma$. Furthermore if $r \in\{-1,0,1\}$ was in $\hat{\mathcal{N}}$, the weight on the edge between $g$ and its successor gate $g$, we assign to this edge the weight $c \cdot r$. Note that $g^{\prime}$ receives in this way from $g$ the same input as in $\hat{\mathcal{N}}\left[c^{\prime}\right]^{\beta^{\prime}}$ (for every circuit input). Assume now that $\alpha_{1}^{\prime}, \ldots, \alpha_{m}^{\prime}$ are the weights that the incoming edges of $g^{\prime}$ get assigned in this way, that $\alpha_{0}^{\prime}$ is the bias of $g^{\prime}$ in the assignment $z^{\prime}=\left\langle\beta^{\prime}, c^{\prime}\right\rangle$, and that $c^{\prime}>0$ is the scaling factor of $g^{\prime}$ in $\hat{\mathcal{N}}\left[c^{\prime}\right]^{\beta^{\prime}}$. Then we assign the new weights $\frac{\alpha_{1}^{\prime}}{c^{\prime}}, \ldots, \frac{\alpha_{m}^{\prime}}{c^{\prime}}$ and the new bias $\frac{\alpha_{0}^{\prime}}{c^{\prime}}$ to $g^{\prime}$, and we multiply the weight on the outgoing edge from $g^{\prime}$ by $c^{\prime}$.

By construction we have that $\forall x \in \mathbf{R}^{n}\left(\mathcal{N}^{\alpha^{\prime}}(x)=\hat{\mathcal{N}}\left[c^{\prime}\right]^{\beta^{\prime}}(x)\right)$; hence $\forall x \in$ $\mathbf{Q}_{a}^{n}\left(\mathcal{N}^{\alpha^{\prime}}(x)=\mathcal{N}^{\alpha}(x)\right)$

\section{Upper bounds for neural nets with piecewise-polynomial activation functions.}

THEOREM 3.1. Consider an arbitrary array $\left(\mathcal{N}_{n}\right)_{n \in N}$ of high-order network architectures $\mathcal{N}_{n}$ of depth $O(1)$ with $n$ inputs and $O\left(n^{O(1)}\right)$ gates in which the gate function $\gamma^{g}$ of each gate $g$ is piecewise-polynomial of degree $O(1)$ with $O\left(n^{O(1)}\right)$ polynomial pieces, with arbitrary reals as architectural parameters.

Then there exists an array $\left(\hat{\mathcal{N}}_{n}\right)_{n \in N}$ of first-order network architectures $\hat{\mathcal{N}}_{n}$ of depth $O(1)$ with $n$ inputs and $O\left(n^{O(1)}\right)$ gates such that each gate $g$ in $\hat{\mathcal{N}}_{n}$ uses as its activation function the Heaviside function $\mathcal{H}$ (i.e., $g$ is a linear threshold gate) and such that for each assignment $\alpha_{n}$ of arbitrary reals to the programmable parameters in $\mathcal{N}_{n}$ there is an assignment $\tilde{\alpha}_{n}$ of $O\left(n^{O(1)}\right)$ numbers from $\{-1,0,1\}$ to the programmable parameters in $\hat{\mathcal{N}}_{n}$ such that $\forall x \in\{0,1\}^{n}\left(\mathcal{N}_{n}^{\alpha_{n}}(x)=\tilde{\mathcal{N}}_{n}^{\tilde{\alpha}_{n}}(x)\right)$.

Hence for any assignment $\left(\alpha_{n}\right)_{n \in N}$ of real-valued parameters the Boolean functions that are computed by $\left(\mathcal{N}_{n}^{\alpha_{n}}\right)_{n \in N}$ are in $T C^{0}$. In particular, VC dimension $\left(\mathcal{N}_{n},\{0,1\}^{n}\right)=O\left(n^{O(1)}\right)$.

Remark 3.2.

(a) The proof of Theorem 3.1 shows that one can replace in its claim the Boolean domain $\{0,1\}^{n}$ by $\{-K, \ldots, K\}^{n}$ for any $K \in \mathbf{N}$.

(b) Theorem 3.1 yields no bound for the computational power of neural nets with the activation function $\sigma(y)=1 /\left(1+e^{-y}\right)$. However it provides bounds for 
the case where the activation functions are spline approximations to $\sigma$ of arbitrarily high degree $d$, provided that $d \in \mathbf{N}$ is fixed.

Proof of Theorem 3.1. This proof is quite long and involved, even for the simplest nonlinear case where the activation functions consist of two polynomial pieces of degree 2. Note that in contrast to the model in [SS] the magnitude of the given weights in $\mathcal{N}_{n}$ may grow arbitrarily fast as a function of $n$.

We first note that one can eliminate all nonlinear polynomials $\mathbf{Q}^{g}$ as arguments of activation functions by introducing intermediate gates with linear gate inputs and quadratic activation functions. One exploits here the obvious fact that $y \cdot z=\frac{1}{2}((y+$ $\left.z)^{2}-y^{2}-z^{2}\right)$. In this way one can transform the given network architectures into first-order network architectures which still satisfy the assumptions of Theorem 3.1. One should note, however, that this transformation does not affect the degrees of polynomial pieces in the activation functions.

Subsequently we transform each given network architecture $\mathcal{N}_{n}$ into a normal form $\hat{\mathcal{N}}_{n}$ of constant depth and size $O\left(n^{O(1)}\right)$ in which all gates $g$ have fan-out $\leq 1$ and in which all gates $g$ use as activation functions $\gamma^{g}$ piecewise-polynomial functions of the following special type: $\gamma^{g}$ consists of up to three pieces, of which at most one is not identically 0 and in which the nontrivial piece outputs the constant 1 or computes a power $y \mapsto y^{k}$ (where $k \in \mathbf{N}$ satisfies $k=O(1)$ ). The preceding "normalization" of activation functions is easy to achieve, since every activation function of a gate in $\mathcal{N}_{n}$ can be written as linear combination of activation functions of this normalized type. The transformation from $\mathcal{N}_{n}$ to $\hat{\mathcal{N}}_{n}$ can be carried out in such a way that for every assignment $\alpha_{n}$ of real values to the programmable parameters of $\mathcal{N}_{n}$ there exists an assignment $\beta_{n}$ of real numbers to the programmable parameters of $\hat{\mathcal{N}}_{n}$ such that

$$
\forall x \in\{0,1\}^{n}\left(\mathcal{N}_{n}^{\alpha_{n}}(x)=\hat{\mathcal{N}}_{n}^{\beta}{ }^{\beta}(x)\right),
$$

and such that any strict inequality " $s_{1}<s_{2}$ " that arises in the computation of $\hat{\mathcal{N}}_{n}^{\beta}{ }^{\beta}$ for some input $x \in\{0,1\}^{n}$ (when one compares some subresult of that computation with a threshold of the activation function of some gate or with the outer threshold of $\hat{\mathcal{N}}_{n}^{\beta}{ }^{\beta}$ ) can be replaced by the stronger inequality " $s_{1}+1 \leq s_{2}$."

It would also be possible to push all nontrivial weights to the gates on level 1 in correspondence to the construction in the proof of Theorem 2.1. However, in the present context this additional operation does not eliminate nonlinear conditions on the weights. Assume for example that $g$ is a gate on level 1 with input $\alpha_{1} x_{1}+\alpha_{2} x_{2}$ and activation function $\gamma^{g}(y)=y^{2}$. Then this gate $g$ outputs $\alpha_{1}^{2} x_{1}^{2}+2 \alpha_{1} \alpha_{2} x_{1} x_{2}+\alpha_{2}^{2} x_{2}^{2}$. Hence the variables $\alpha_{1}, \alpha_{2}$ will not occur linearly in an inequality which describes the comparison of the output of $g$ with some threshold of a gate at the next level.

Although it does not eliminate nonlinear conditions on the weights if one pushes all weights toward level 1, the resulting network provides some notational advantage because all weights between computation nodes can be treated as constants (with three possible values). This approach has therefore been chosen in [M92] and [M93a]. However, this approach is disadvantageous if one wants to apply the method of this proof in the context of agnostic PAC learning on analog neural nets [M93c]. In this application one has to be able to control the bit-length of the (rational) weights. Therefore, one cannot afford to push all weights toward level 1 , since this may increase the bit-length of weights in an unbounded manner. For example, if one pushes the weight 2 through a gate $g$ with activation function $\gamma^{g}(y)=y^{2}$, then this weight is changed to $\sqrt{ } 2$ (since $\left.2 \gamma^{g}(y)=\gamma^{g}(\sqrt{ } 2 \cdot y)\right)$. 
Since the nonlinearity of the conditions on the weights cannot be eliminated in the same way as for Theorem 2.1, we have to introduce an alternative method. We fix an arbitrary assignment $\beta_{n}$ of real numbers to the programmable parameters of $\hat{\mathcal{N}}_{n}$. We introduce for the system of inequalities $L\left(\hat{\mathcal{N}}_{n}^{\beta}{ }^{\beta},\{0,1\}^{n}\right)$ (that describes the computations of $\hat{\mathcal{N}}_{n}^{\beta}$ for all inputs $x \in\{0,1\}^{n}$ ) new variables $v$ for all nontrivial parameters in $\hat{\mathcal{N}}_{n}^{\beta}$ (i.e., for the weights and bias of each gate $g$, for the outer threshold $T_{\text {out }}$, and for the thresholds $t_{1}^{g}, t_{2}^{g}$ of each gate $g$ ). In addition we introduce new variables for all products of such parameters that arise in the computation of $\hat{\mathcal{N}}_{n}^{\beta}$. We have to keep the inequalities linear in order to apply Lemma 2.4. Hence we cannot demand in these inequalities that the value of the variable $v_{v_{1}^{g}, v_{2}^{g}}$ (that represents the product of $\alpha_{1}^{g}$ and $\alpha_{2}^{g}$ ) is the product of the values of the variables $v_{1}^{g}$ and $v_{2}^{g}$ (that represent the weights $\alpha_{1}^{g}$, respectively, $\alpha_{2}^{g}$ ). We solve this problem by describing in detail in the linear inequalities $L\left(\hat{\mathcal{N}}_{n}^{\beta}{ }^{n},\{0,1\}^{n}\right)$ which role the product of $\alpha_{1}^{g}$ and $\alpha_{2}^{g}$ plays in the computations of $\hat{\mathcal{N}}_{n}^{\beta}{ }^{n}$ for inputs from $\{0,1\}^{n}$. It turns out that this can be done in such a way that it does not matter whether a solution $\mathcal{A}$ of $L\left(\hat{\mathcal{N}}_{n}^{\beta}{ }^{\beta}\right.$, $\left.\{0,1\}^{n}\right)$ assigns to the variable $v_{v_{1}^{g}, v_{2}^{g}}$ a value $\mathcal{A}\left(v_{v_{1}^{g}}, v_{2}^{g}\right)$ that is equal to the product of the values $\mathcal{A}\left(v_{1}^{g}\right)$ and $\mathcal{A}\left(v_{2}^{g}\right)$ (that are assigned by $\mathcal{A}$ to the variables $v_{1}^{g}$ and $v_{2}^{g}$ ). In any case $\mathcal{A}\left(v_{v_{1}^{g}, v_{2}^{g}}\right)$ is forced to behave like the product of $\mathcal{A}\left(v_{1}^{g}\right)$ and $\mathcal{A}\left(v_{2}^{g}\right)$ in the computations of $\hat{\mathcal{N}}_{n}^{\beta}{ }^{\beta}$.

We would like to emphasize that the parameters $\beta_{n}$ do not occur as constants in the system $L\left(\hat{\mathcal{N}}_{n}^{\beta}{ }^{n},\{0,1\}^{n}\right)$ of inequalities. They are also replaced by variables. The reason the real-valued parameters $\beta$ occur nevertheless in our notation $L\left(\hat{\mathcal{N}}_{n}^{\beta}{ }^{\beta}\right.$, $\{0,1\}^{n}$ ) of inequalities is the following. These inequalities consist of conditions which demand that for any input $x \in\{0,1\}^{n}$ the computation on the neural net proceeds exactly as for the parameter assignment $\beta_{n}$ (i.e., the same inequalities with thresholds of the piecewise-polynomial activation functions are satisfied and the same pieces of the activation functions are used at each gate as in the computation with parameter assignment $\beta_{n}$ ).

Before we present the formal definitions and proofs, we give a high-level description of the proof idea and the purpose of the formal definitions. These preceding informal remarks should be real interactively with the subsequent formal part.

In more abstract terms, one may view any solution $\mathcal{A}$ of $L\left(\hat{\mathcal{N}}_{n}^{\beta}{ }^{\beta},\{0,1\}^{n}\right)$ as a model of a certain linear fragment $L\left(\hat{\mathcal{N}}_{n}^{\beta}{ }^{\beta},\{0,1\}^{n}\right)$ of the theory of the role of the parameters $\beta_{n}$ in the computations of $\hat{\mathcal{N}}_{n}^{\beta}{ }^{\beta}$ on inputs from $\{0,1\}^{n}$. Such a model $\mathcal{A}$ (which will be given by Lemma 2.4) may be viewed as some type of nonstandard model of the theory of computations of $\hat{\mathcal{N}}_{n}^{\beta}$, since it replaces products of weights by values that one might call nonstandard products. Such a nonstandard model $\mathcal{A}$ does not provide a new assignment of (small) weights to the network architecture $\hat{\mathcal{N}}_{n}$, only to a nonstandard version $\mathcal{M}_{n}^{\mathcal{A}}$ of the neural net $\hat{\mathcal{N}}_{n}^{\beta}{ }^{n}$. However the linear fragment $L\left(\hat{\mathcal{N}}_{n}^{\beta}{ }^{\beta},\{0,1\}^{n}\right)$ can be chosen in such a way that $\mathcal{M}_{n}^{\mathcal{A}}$ computes the same Boolean function as $\hat{\mathcal{N}}_{n}^{\beta}{ }^{\beta}$. Furthermore, if $\mathcal{A}$ consists of a solution with rational numbers as given by Lemma 2.4 , then $\mathcal{M}_{n}^{\mathcal{A}}$ can be simulated by a constant-depth polynomialsize Boolean circuit whose gates $g$ are all MAJORITY-gates (i.e., $g\left(y_{1}, \ldots, y_{m}\right)=1$ if $\sum_{i=1}^{m} y_{i} \geq m / 2$; otherwise $\left.g\left(y_{1}, \ldots, y_{m}\right)=0\right)$. This implies that the Boolean functions 
that are computed by $\left(\mathcal{M}_{n}^{\mathcal{A}}\right)_{n \in \mathbf{N}}$ are in $\mathrm{TC}^{0}$. However, by construction these are the same Boolean functions that are computed by $\left(\mathcal{N}_{n}^{\alpha_{n}}\right)_{n \in \mathbf{N}}$.

We will now describe the details of the previously sketched proof of Theorem 3.1. We will simply write $\mathcal{N}$ instead of $\hat{\mathcal{N}}_{n}^{\beta}{ }^{\beta}$ (where $\beta_{n}$ is some assignment of real numbers to the programmable parameters of the network architecture $\hat{\mathcal{N}}_{n}$ ). We will define for each gate $g$ in $\mathcal{N}$ by induction on the depth of $g$,

(1) in Definition 3.3 a set $V^{g}$ of variables and a set $M^{g}$ of formal terms that are needed to describe the operation of gate $g$.

[The intuition is here that one writes for any network input $x$ the output of $g$ as a sum of products (of programmable parameters, of architectural parameters, and of components of $x$ ). Which of these terms will occur for a specific circuit input $x$ will depend on the course of the computation in $\mathcal{N}$ up to gate $g$ : for different inputs the involved gates may use different pieces of their activation function. The set $M^{g}$ contains a separate formal term for each product that may possibly occur in this sum. Each term in $M^{g}$ consists of a variable $w \in V^{g}$ (that represents a programmable or architectural parameter of $\mathcal{N}$ or some product of these) and of a product $P \equiv \pm \boldsymbol{x}_{1}^{j_{1}} \cdot \ldots \cdot \boldsymbol{x}_{n}^{j_{n}}$ of input variables $\boldsymbol{x}_{1}, \ldots, \boldsymbol{x}_{n}$.]

(2) in Definition 3.4 for any fixed network input $x \in \mathbf{R}^{n}$ a set $L^{g}(x)$ of linear inequalities associated with gate $g$ (with variables from $V^{\mathcal{N}}:=\cup\left\{V^{g^{\prime}} \mid g^{\prime}\right.$ is a gate of $\mathcal{N}\}$ ) that hold for the computation of $\mathcal{N}$ on input $x$ if all formal terms $t \in M^{g}$ are replaced by their actual value $W(t, x)$ for the given parameter assignment in $\mathcal{N}$. We also define in Definition 3.4 a set $S^{g}(x)$ of formal terms whose sum represents the input of $g$ and a set $T^{g}(x) \subseteq M^{g}$ of formal terms whose sum represents the output of $g$ for circuit input $x$.

$\left[L^{g}(x)\right.$ specifies in particular which piece of $\gamma^{g}$ is used by gate $g$ for network input $x$.]

(3) in Definition 3.6 for any input set $S \subseteq \mathbf{R}^{n}$, any solution $\mathcal{A}$ of the resulting system $L(\mathcal{N}, S):=\cup\left\{L^{g}(x) \mid x \in S\right.$ and $g$ is a gate in $\left.\mathcal{N}\right\}$ of linear inequalities, and any term $t \in M^{g}$ a network architecture $\mathcal{M}_{g, t}^{\mathcal{A}}$ that decides for any network input $x \in S$ whether $t$ occurs as a summand in the output of $g$ in $\mathcal{N}$.

[For any input $x \in S$ the network architectures $\left(\mathcal{M}_{g, t}^{\mathcal{A}}\right)_{t \in M^{g}}$ together compute the characteristic function of the set $T^{g}(x) \subseteq M^{g}$ which represents the output of gate $g$ in $\mathcal{N}$. In this way one can replace in a recursive manner the analog computations in $\mathcal{N}$ by digital manipulations of formal terms, with "nonstandard products" of weights in place of real products.]

One verifies in Lemma 3.5 that $L(\mathcal{N}, S)$ describes correctly the role of the parameters $\beta_{n}$ in the computations of $\mathcal{N}:=\hat{\mathcal{N}}_{n}^{\beta}{ }^{n}$ for inputs $x \in S$. Unfortunately, $L(\mathcal{N}, S)$ does not provide a complete description of the properties of the parameters $\beta_{n}$ in these computations, since it represents only a linear fragment of their theory. Nevertheless, one can prove with the help of Lemmas 3.7 and 3.8 that for any solution $\mathcal{A}$ of $L(\mathcal{N}, S)$ the network architectures $\mathcal{M}_{g, t}^{\mathcal{A}}$ carry out a truthful simulation of the corresponding initial segments of $\mathcal{N}$.

We would like to point out a difference to the proof of Theorem 2.1 regarding the treatment of architectural parameters. In the proof of Theorem 3.1 the programmable parameters $\alpha_{n}$ of $\mathcal{N}_{n}^{\alpha_{n}}$ and the architectural parameters of the given network architecture $\mathcal{N}_{n}$ (the thresholds of activation functions $\gamma^{g}$ and the coefficients of the polynomial pieces of $\gamma^{g}$ ) are all changed simultaneously in the transformation to $\hat{\mathcal{N}}_{n}^{\beta}$. Consequently, $\beta_{n}$ denotes the values of all nontrivial parameters in $\hat{\mathcal{N}}_{n}^{\beta}{ }^{\beta}$ (i.e., of all 
programmable and architectural parameters). As a consequence of this treatment of parameters one can allow in the given network architectures $\mathcal{N}_{n}$ of Theorem 3.1 arbitrary reals as architectural parameters (i.e., for the thresholds and coefficients of the polynomial pieces of the given activation functions $\gamma^{g}$ ).

We refer to an analog network architecture $\mathcal{N}$ with the properties of $\hat{\mathcal{N}}_{n}^{\beta}{ }^{\beta}$ as a network architecture in normal form. This means that $\mathcal{N}$ is a first-order network architecture whose gates have fan-out $\leq 1$; all gates $g$ in $\mathcal{N}$ use as activation function $\gamma^{g}$ a piecewise-polynomial function that consists of three pieces, of which at most one piece is not identically 0 and in which the nontrivial piece (if it exists) outputs the constant 1 or computes a power $y \mapsto y^{k}$ for some $k \in \mathbf{N}$.

In order to simplify our notation, we assume that for a network architecture $\mathcal{N}$ in normal form the nontrivial piece of the activation function $\gamma^{g}$ of each gate $g$ is defined over a half-open interval $\left[t_{1}^{g}, t_{2}^{g}\right)$ with certain reals $t_{1}^{g}<t_{2}^{g}$. It is easy to see that the subsequent proof can also be carried out without this simplifying assumption. We also assume without loss of generality (w.l.o.g.) that $\mathcal{N}$ is leveled, i.e., each gate $g$ in $\mathcal{N}$ has the property that all paths in $\mathcal{N}$ from an input node to $g$ have the same length.

Definition 3.3. Assume that $\mathcal{N}$ is a network architecture in normal form with $n$ input variables $x_{1}, \ldots, x_{n}$, where arbitrary reals have been assigned to all parameters of $\mathcal{N}$. We define by induction on the depth of $g$ for each gate $g$ in $\mathcal{N}$ a set $V^{g}$ of variables, a value $W(v)$ for each variable $v \in V^{g}$ (that arises from the assignment $\beta_{n}$ in $\hat{\mathcal{N}}_{n}^{\beta}{ }^{n}:=\mathcal{N}$ ), and a set $M^{g}$ of (formal) terms. Each element of $M^{g}$ is of the form $v \cdot P$, where $v \in V^{g}$ is a variable and $P$ is some formal polynomial term of the form $\pm \boldsymbol{x}_{1}^{j_{1}} \cdot \ldots \cdot \boldsymbol{x}_{n}^{j_{n}}$, with $j_{1}, \ldots, j_{n} \in \boldsymbol{N}$. The here-occurring formal variables $\boldsymbol{x}_{1}, \ldots, \boldsymbol{x}_{n}$ for the input components should be distinguished from the concrete values $x_{1}, \ldots, x_{n} \in \boldsymbol{R}$ for these variables that are considered later (starting in Definition 3.4).

We consider first the case where $g$ has depth 1 . If $\gamma^{g}$ gives on its nontrivial piece $\left[t_{1}^{g}, t_{2}^{g}\right)$ the constant 1 as output, we set

$$
V^{g}:=\left\{v_{0}^{g}, \ldots, v_{n}^{g}\right\} \cup\left\{v_{\text {const }}^{g}\right\} \cup\left\{v_{\mathrm{I}}^{g}, v_{\mathrm{II}}^{g}\right\} \text { and } M^{g}:=\left\{v_{\text {const }}^{g}\right\} .
$$

We define $W\left(v_{i}^{g}\right):=\alpha_{i}^{g}$ for $i=0, \ldots, n, W\left(v_{\text {const }}^{g}\right):=\alpha^{g}, W\left(v_{\mathrm{I}}^{g}\right):=t_{1}^{g}$, and $W\left(v_{\mathrm{II}}^{g}\right):=$ $t_{2}^{g}\left(\alpha_{1}^{g}, \ldots, \alpha_{n}^{g}\right.$ are the weights and $\alpha_{0}^{g}$ is the bias of gate $g$ in $\mathcal{N}, \alpha^{g}$ is the weight on the edge that leaves $g$, and $t_{1}^{g}, t_{2}^{g}$ are the thresholds of the activation function $\left.\gamma^{g}\right)$. In the other case $\gamma^{g}$ computes a power $y \mapsto y^{k}$ on its nontrivial piece. Then we introduce for each ktuple $\left\langle w_{1} \cdot P_{1}, \ldots, w_{k} \cdot P_{k}\right\rangle \in\left(\left\{v_{0}^{g}\right\} \cup\left\{v_{1}^{g} \cdot \boldsymbol{x}_{1}, \ldots, v_{n}^{g} \cdot \boldsymbol{x}_{n}\right\}\right)^{k}$ a new variable $v_{w_{1}, \ldots, w_{k}}^{g}$ in $V^{g}$ and a term $v_{w_{1}, \ldots, w_{k}}^{g} \cdot \prod_{i=1}^{k} P_{i}$ in $M^{g}$. We assume here that a formal multiplication $P \cdot P^{\prime}$ for formal terms $P, P^{\prime}$ of the form $\pm x_{1}^{j_{1}} \cdot \ldots \cdot \boldsymbol{x}_{n}^{j_{n}}$ is defined in the obvious way. We define

$$
V^{g}:=\left\{v_{0}^{g}, \ldots, v_{n}^{g}\right\} \cup\left\{v_{\mathrm{I}}^{g}, v_{\mathrm{II}}^{g}\right\} \cup\left\{v_{w_{1}, \ldots, w_{k}}^{g} \mid\left\langle w_{1}, \ldots, w_{k}\right\rangle \in\left\{v_{0}^{g}, \ldots, v_{n}^{g}\right\}^{k}\right\} .
$$

We set $W\left(v_{w_{1}, \ldots, w_{k}}^{g}\right):=\alpha^{g} \cdot \prod_{i=1}^{k} W\left(w_{i}\right)$, and we define $W(v)$ for the other variables as before. We define

$$
M^{g}:=\left\{v_{w_{1}, \ldots, w_{k}}^{g} \cdot \prod_{i=1}^{k} P_{i} \mid\left\langle w_{1} \cdot P_{1}, \ldots, w_{k} \cdot P_{k}\right\rangle \in\left(\left\{v_{0}^{g}\right\} \cup\left\{v_{1}^{g} \cdot \boldsymbol{x}_{1}, \ldots, v_{n}^{g} \cdot \boldsymbol{x}_{n}\right\}\right)^{k}\right\} .
$$

[The terms in $M^{g}$ denote the summands that one gets from the output $\left(\alpha_{0}^{g}+\sum_{i=1}^{m} \alpha_{i}^{g}\right.$. $\left.x_{i}\right)^{k}$ of $\gamma^{g}$ by multiplying this output with the weight $\alpha^{g}$ on the next edge and then rewriting it as a sum of products.] 
We now consider the case where $g$ is a gate on level $l+1$, with edges from the gates $g_{1}, \ldots, g_{m}$ on level l leading into $g$. Assume that $\alpha_{1}^{g}, \ldots, \alpha_{m}^{g}$ are in $\mathcal{N}$, the weights on these edges, that $\alpha^{g}$ is the weight on the edge out of $g$, and that $\alpha_{0}^{g}$ is the bias of $g$. If $g$ is an output gate (i.e., $g$ has fan-out 0 ) then we set $\alpha^{g}:=1$. If $\gamma^{g}$ outputs the constant 1 on its nontrivial piece, we set

$$
V^{g}:=\left\{v_{0}^{g}, v_{\text {const }}^{g}\right\} \cup\left\{v_{\mathrm{I}}^{g}, v_{\mathrm{II}}^{g}\right\} \text { and } M^{g}:=\left\{v_{\text {const }}^{g}\right\} .
$$

We set $W\left(v_{0}^{g}\right):=\alpha_{0}^{g}, W\left(v_{\text {const }}^{g}\right):=\alpha^{g}, W\left(v_{\mathrm{I}}^{g}\right):=t_{1}^{g}$, and $W\left(v_{\mathrm{II}}^{g}\right):=t_{2}^{g}$. If $\gamma^{g}$ computes the power $y \mapsto y^{k}$ on its nontrivial piece, we introduce for each ktuple

$$
\left\langle w_{1} \cdot P_{1}, \ldots, w_{k} \cdot P_{k}\right\rangle \in\left(\left\{v_{0}^{g}\right\} \cup \bigcup_{j=1}^{m} M^{g_{j}}\right)^{k}
$$

a new variable $v_{w_{1}, \ldots, w_{k}}^{g}$ in $V^{g}$ and a term $v_{w_{1}, \ldots, w_{k}}^{g} \cdot \prod_{i=1}^{k} P_{i}$ in $M^{g}$. Thus we set

$$
\begin{aligned}
V^{g}:=\left\{v_{0}^{g}\right\} & \cup\left\{v_{\mathrm{I}}^{g}, v_{\mathrm{II}}^{g}\right\} \\
& \cup\left\{v_{w_{1}, \ldots, w_{k}}^{g} \mid\left\langle w_{1} \cdot P_{1}, \ldots, w_{k} \cdot P_{k}\right\rangle \in\left(\left\{v_{0}^{g}\right\} \cup \bigcup_{j=1}^{m} M^{g_{j}}\right)^{k},\right.
\end{aligned}
$$

for arbitrary polynomial terms $P_{1}, \ldots, P_{k}$ and variables

$$
\left.w_{i} \in\left(\left\{v_{0}^{g}\right\} \cup \bigcup_{j=1}^{m} V^{g_{j}}\right)\right\} .
$$

$t_{2}^{g}$.

We define $W\left(v_{w_{1}, \ldots, w_{k}}^{g}\right):=\alpha^{g} \cdot \prod_{i=1}^{k} W\left(w_{i}\right), W\left(v_{0}^{g}\right):=\alpha_{0}^{g}, W\left(v_{\mathrm{I}}^{g}\right):=t_{1}^{g}, W\left(v_{\mathrm{II}}^{g}\right):=$ We set

$$
M^{g}:=\left\{v_{w_{1}, \ldots, w_{k}}^{g} \cdot \prod_{i=1}^{k} P_{i} \mid\left\langle w_{1} \cdot P_{1}, \ldots, w_{k} \cdot P_{k}\right\rangle \in\left(\left\{v_{0}^{g}\right\} \cup \bigcup_{j=1}^{m} M^{g_{j}}\right)^{k}\right\} .
$$

[The argument of $\gamma^{g}$ is a sum of $\alpha_{0}^{g}$ and of summands that are denoted by terms in $\cup_{j=1}^{m} M^{g_{j}}$. Hence the terms in $M^{g}$ correspond to the summands that one gets by multiplying the output of $\gamma^{g}$ with the weight on the next edge and then rewriting this product as a sum of products by multiplying out.]

Finally, for the output gate $g_{\text {out }}$ of $\mathcal{N}$, we place into $V^{g}$ in addition the variable $v^{g_{\text {out }}}$. We define $W\left(v^{g_{\text {out }}}\right)$ as the value of the outer threshold of $\mathcal{N}$.

DEFINITION 3.4. Assume that $\mathcal{N}$ is a network architecture in normal form with $n$ input variables and some fixed assignment of reals to its parameters. Let $x \in \boldsymbol{R}^{n}$ be a fixed input for $\mathcal{N}$. We define for each gate $g$ in $\mathcal{N}$ by simultaneous induction on the depth of $g$

(1) a set $L^{g}(x)$ of inequalities (that are linear in the variables from $V^{G}$ ),

(2) a set $S^{g}(x)$ of formal terms (whose sum represents the argument of $\gamma^{g}$ for network input $x)$,

(3) a set $T^{g}(x) \subseteq M^{g}$ (whose sum represents the output of $g$ for network input $x$ after multiplication with the weight on the next edge).

Since $x$ is now a fixed element of $\boldsymbol{R}^{n}$, one can assign a specific value $W(P, x) \in \boldsymbol{R}$ to each term $P$ of the form $\pm \boldsymbol{x}_{1}^{j_{1}} \cdot \ldots \cdot \boldsymbol{x}_{n}^{j_{n}}$ that occurs in a formal term of the preceding 
definition. Hence one can assign to any formal term $t=v \cdot P$ (that belongs to some $\left.M^{g}\right)$ a specific value $W(t, x):=W(v) \cdot W(P, x)$. For a set $S$ of formal terms we define $W(S, x):=\sum_{t \in S} W(t, x)$. For the case $S=\phi$ we set $W(\phi, x):=0$.

The value $W(t, x)$ of a formal term $t$ reflects the value of this term for network input $x$ under the fixed parameter assignment in $\mathcal{N}$. These values $W(t, x)$ are needed for the definition of the systems $L^{g}(x)$ and $L(\mathcal{N}, x)$ of linear inequalities that describe the computation of $\mathcal{N}$.

If $g$ has depth 1 , then we define

$$
S^{g}(x):=\left\{v_{0}^{g}\right\} \cup\left\{v_{i}^{g} \cdot \boldsymbol{x}_{i} \mid i=1, \ldots, n\right\} .
$$

Assume that $g$ is a gate on level $l+1$ with edges from gates $g_{1}, \ldots, g_{m}$ on level $l$ leading into $g$. Then we set

$$
S^{g}(x):=\left\{v_{0}^{g}\right\} \cup \bigcup_{j=1}^{m} T^{g_{j}}(x) .
$$

We define $L^{g}(x)$ and $T^{g}(x)$ as follows for any gate $g$ in $\mathcal{N}$. If $W\left(S^{g}(x), x\right)<t_{1}^{g}$, then $L^{g}(x)$ contains the inequality $\left[\sum S^{g}(x)+1\right]_{x}[x] \leq v_{\mathrm{I}}^{g}$. If $W\left(S^{g}(x), x\right) \geq t_{2}^{g}$, then $L^{g}(x)$ contains the inequality $\left[\sum S^{g}(x)\right]_{x}[x] \geq v_{\mathrm{II}}^{g}$. In either case we set $T^{g}(x):=\phi$.

[We use here and in the following the notation $[H]_{x}[x]$ for any sum $H$ of formal terms to indicate that each variable $\boldsymbol{x}_{i}$ in $H$ is replaced by the value of the $i$ th coordinate $x_{i}$ of the concrete input $x \in \mathbf{R}^{n}$. Note that the only variables that are left in $[H]_{x}[x]$ are the variables of the form $v_{\text {const }}^{g}, v_{i}^{g}$, or $v_{w_{1}, \ldots, w_{k}}^{g}$. This substitution is necessary to make sure that the only variables that occur in the resulting system $L(\mathcal{N}, S)$ of linear inequalities are of this type or are variables of the form $v_{\mathrm{I}}^{g}, v_{\mathrm{II}}^{g}$.]

If $t_{1}^{g} \leq W\left(S^{g}(x), x\right)<t_{2}^{g}$, then $L^{g}(x)$ contains the inequalities $v_{\mathrm{I}}^{g} \leq\left[\sum S^{g}(x)\right]_{x}[x]$ and $\left[\sum S^{g}(x)+1\right]_{x}[x] \leq v_{\mathrm{II}}^{g}$. If $\gamma^{g}$ gives on its nontrivial piece a constant $a^{g}$ as output, we set in this case $T^{g}(x):=\left\{v_{\text {const }}^{g}\right\}$. If $\gamma^{g}$ computes on its nontrivial piece a power $y \mapsto y^{k}$, we set

$$
T^{g}(x):=\left\{v_{w_{1}, \ldots, w_{k}}^{g} \cdot \prod_{i=1}^{k} P_{i} \mid\left\langle w_{1} \cdot P_{1}, \ldots, w_{k} \cdot P_{k}\right\rangle \in\left(\left\{v_{0}^{g}\right\} \cup\left\{v_{1}^{g} \cdot \boldsymbol{x}_{1}, \ldots, v_{n}^{g} \cdot \boldsymbol{x}_{n}\right\}\right)^{k}\right\}
$$

if $g$ has depth 1 , and in the general case

$$
T^{g}(x):=\left\{v_{w_{1}, \ldots, w_{k}}^{g} \cdot \prod_{i=1}^{k} P_{i} \mid\left\langle w_{1} \cdot P_{1}, \ldots, w_{k} \cdot P_{k}\right\rangle \in\left(\left\{v_{0}^{g}\right\} \cup \bigcup_{j=1}^{m} T^{g_{j}}(x)\right)^{k}\right\} .
$$

Finally, if $g$ is the output gate $g_{\text {out }}$ of $\mathcal{N}$ and $W\left(T^{g_{\text {out }}}(x), x\right)<W\left(v^{g_{\text {out }}}\right)$, we add to $L^{g_{\text {out }}}(x)$ also the inequality $\left[\sum T^{g_{\text {out }}}(x)+1\right]_{x}[x] \leq v^{g_{\text {out }}}$. If $W\left(T^{g_{\text {out }}}(x), x\right) \geq W\left(v^{g_{\text {out }}}\right)$, we add to $L^{g_{\text {out }}}(x)$ also the inequality $\left[\sum T^{g_{\text {out }}}(x)\right]_{x}[x] \geq v^{g_{\text {out }}}$.

We define

$$
L(\mathcal{N}, x):=\bigcup\left\{L^{g}(x) \mid g \text { is a gate of } \mathcal{N}\right\}
$$

and for $S \subseteq \boldsymbol{R}^{n}$

$$
L(\mathcal{N}, S):=\bigcup\{L(\mathcal{N}, x) \mid x \in S\}
$$

The following lemma verifies that for any $x \in \boldsymbol{R}^{n}$ the system $L(\mathcal{N}, x)$ of inequalities provides a truthful description of the computation of $\mathcal{N}$ for input $x$. 
Lemma 3.5. Assume that $\mathcal{N}$ is a network architecture in normal form with $n$ input variables and some arbitrary assignment to its parameters and that $x \in \boldsymbol{R}^{n}$ is an arbitrary concrete input.

Then we have for any gate $g$ in $\mathcal{N}$ that $W\left(S^{g}(x), x\right)$ is the input and $W\left(T^{g}(x), x\right)$ is the output of gate $g$ (multiplied with the weight on the next edge) in the computation of $\mathcal{N}$ for input $x$. Furthermore,

$$
\begin{aligned}
& W\left(S^{g}(x), x\right)<t_{1}^{g} \Leftrightarrow "\left[S^{g}(x)+1\right]_{x}[x] \leq v_{\mathrm{I}}^{g "} \in L(\mathcal{N}, x) \\
& W\left(S^{g}(x), x\right)<t_{2}^{g} \Leftrightarrow "\left[S^{g}(x)+1\right]_{x}[x] \leq v_{\mathrm{II}}^{g} " \in L(\mathcal{N}, x) \\
& W\left(S^{g}(x), x\right) \geq t_{1}^{g} \Leftrightarrow "\left[S^{g}(x)\right]_{x}[x] \quad \geq v_{\mathrm{I}}^{g "} \in L(\mathcal{N}, x) \\
& W\left(S^{g}(x), x\right) \geq t_{2}^{g} \Leftrightarrow "\left[S^{g}(x)\right]_{x}[x] \quad \geq v_{\mathrm{II}}^{g "} \in L(\mathcal{N}, x) .
\end{aligned}
$$

Proof. The claim about $L(\mathcal{N}, x)$ follows immediately from the definition of $L(\mathcal{N}, x)$ in Definition 3.4.

One shows by induction on $g$ that for any network input $x$ the input of $g$ in $\mathcal{N}$ is equal to $W\left(S^{g}(x), x\right)$, and the output of $g$ in $\mathcal{N}$ (after multiplication with the weight on the next edge) is equal to $W\left(T^{g}(x), x\right)$.

If $g$ is of depth 1 then we have by the definition of $S^{g}(x)$ in Definition 3.4 and by the definition of the values $W(t, x)$ for terms $t$ in Definition 3.3 that $W\left(S^{g}(x), x\right)=$ $\alpha_{0}^{g}+\sum_{i=1}^{n} \alpha_{i}^{g} \cdot x_{i}$, where $\alpha_{1}, \ldots, \alpha_{n}^{g}$ are the weights and $\alpha_{0}^{g}$ is the bias of gate $g$ in $\mathcal{N}$ under the given parameter assignment in $\mathcal{N}$. Hence $W\left(S^{g}(x), x\right)$ is equal to the input of $g$ in $\mathcal{N}$ for network input $x$. Furthermore if $\alpha_{0}^{g}+\sum_{i=1}^{n} \alpha_{i}^{g} \cdot x_{i}<t_{1}^{g}$ or $\alpha_{0}^{g}+\sum_{i=1}^{n} \alpha_{i}^{g} \cdot x_{i} \geq t_{2}^{g}$ then $T^{g}(x)=\phi$; hence $W\left(T^{g}(x), x\right)=0$. If $t_{1}^{g} \leq \alpha_{0}^{g}+\sum_{i=1}^{n} \alpha_{i}^{g} \cdot x_{i}<t_{2}^{g}$ then $W\left(T^{g}(x), x\right)=\alpha^{g}$ if $\gamma^{g}$ outputs the constant 1 on its nontrivial piece (where $\alpha^{g}$ is the weight on the edge out of $g$ ). If $\gamma^{g}$ computes $y \mapsto y^{k}$ on its nontrivial piece, then $W\left(T^{g}(x), x\right)=\alpha^{g} \cdot \sum\left(\left\{\alpha_{0}^{g}\right\} \cup\left\{\alpha_{i}^{g} \cdot x_{i} \mid i=1, \ldots, n\right\}\right)^{k}$. In either case $W\left(T^{g}(x), x\right)$ is equal to the output of $g$ in $\mathcal{N}$ (multiplied with $\alpha^{g}$ ) for network input $x$.

If $g$ is of depth $l+1$ with immediate predecessors $g_{1}, \ldots, g_{m}$ then $W\left(S^{g}(x), x\right)=$ $\alpha_{0}^{g}+\sum_{j=1}^{m} W\left(T^{g_{j}}(x), x\right)$. By induction hypothesis this value is equal to the input of gate $g$ in $\mathcal{N}$ for network input $x$. In the most interesting case, where gate $g$ applies the polynomial piece $y \mapsto y^{k}$ to this input, its output (multiplied with $\alpha^{g}$ ) is equal to

$$
\begin{aligned}
& \alpha^{g} \cdot\left(\alpha_{0}^{g}+\sum_{j=1}^{m} W\left(T^{g_{j}}(x), x\right)\right)^{k} \\
& =\alpha^{g} \cdot \sum\left\{\sum_{i=1}^{k} W\left(w_{i} \cdot P_{i}, x\right) \mid\left\langle w_{1} \cdot P_{1}, \ldots, w_{k} \cdot P_{k}\right\rangle \in\left(\left\{v_{0}^{g}\right\} \cup \bigcup_{j=1}^{m} T^{g_{j}}(x)\right)^{k}\right\} \\
& =\sum\left\{W\left(v_{w_{1}, \ldots, w_{k}}^{g}\right) \cdot \prod_{i=1}^{k} W\left(P_{i}, x\right) \mid\left\langle w_{1} \cdot P_{1}, \ldots, w_{k} \cdot P_{k}\right\rangle \in\left(\left\{v_{0}^{g}\right\} \cup \bigcup_{j=1}^{m} T^{g_{j}}(x)\right)^{k}\right\} \\
& =W\left(T^{g}(x), x\right) .
\end{aligned}
$$


DeFINITION 3.6. Assume that $\mathcal{N}$ is a neural act in normal form with $n$ inputs. Furthermore, assume that $S \subseteq \boldsymbol{R}^{n}$ and $\mathcal{A}: V^{\mathcal{N}} \rightarrow \boldsymbol{R}$ is an arbitrary solution of the system $L(\mathcal{N}, S)$ of inequalities with the variable set $V^{\mathcal{N}}:=\bigcup\left\{V^{g} \mid g\right.$ is a gate in $\left.\mathcal{N}\right\}$.

We define by induction on the depth of gate $g$ in $\mathcal{N}$ for each term $t \in M^{g}$ a first-order network architecture $\mathcal{M}_{g, t}^{\mathcal{A}}$. Together the network architectures $\left(\mathcal{M}_{g, t}^{\mathcal{A}}\right)_{t \in M^{g}}$ mimic the initial segment of $\mathcal{N}$ between the input and gate $g$. The first-order network architecture $\mathcal{M}_{g, t}^{\mathcal{A}}$ consists of gates with activation functions from the class $\{$ Heaviside, $\left.y \mapsto y, y \mapsto y^{2}\right\}$. For any circuit input $x \in S$ the output of the first-order network architecture $\mathcal{M}_{g, t}^{\mathcal{A}}$ will be 1 if $t \in T^{g}(x)$; otherwise it will be 0 .

One associates with each network architecture $\mathcal{M}_{g, t}^{\mathcal{A}}$ for $t \in M^{g}$ of the form $t \equiv$ $v \cdot P$ another network architecture $\tilde{\mathcal{M}}_{g, t}^{\mathcal{A}}$ that outputs for any network input $x \in S$ the real number

$$
\mathcal{A}(t, x):=\left\{\begin{array}{cl}
\mathcal{A}(v) \cdot W(P, x) & \text { if } \mathcal{M}_{g, t}^{\mathcal{A}}(x)=1 \\
0 & \text { if } \mathcal{M}_{g, t}^{\mathcal{A}}(x)=0
\end{array}\right.
$$

The extension from $\mathcal{M}_{g, t}^{\mathcal{A}}$ to $\tilde{\mathcal{M}}_{g, t}^{\mathcal{A}}$ is done in a canonical manner with the help of subcircuits that simulate product gates via the equality $y \cdot z=\frac{1}{2}\left((y+z)^{2}-y^{2}-z^{2}\right)$. Obviously, $\tilde{\mathcal{M}}_{g, t}^{\mathcal{A}}$ just has to compute the product of $\mathcal{A}(v), W(P, x)$ and of the output of $\mathcal{M}_{g, t}^{\mathcal{A}}$ for network input $x$.

The definition of a value $\mathcal{A}(t, x)$ for each term $t$ and each $x \in S$ is extended in a canonical way to arbitrary sets $M$ of terms

$$
\mathcal{A}(M, x):=\sum_{t \in M} \mathcal{A}(t, x), \quad \mathcal{A}(\phi, x):=0
$$

We consider first the case where $g$ has depth 1 . Let $H_{1}^{g}$ be a linear threshold gate that checks whether $\mathcal{A}\left(v_{\mathrm{I}}^{g}\right) \leq \mathcal{A}\left(S^{g}(x), x\right)$, and let $H_{2}^{g}$ be a linear threshold gate that checks whether $\mathcal{A}\left(S^{g}(x), x\right)+1 \leq \mathcal{A}\left(v_{\mathrm{II}}^{g}\right)$. For each term $t \in M^{g}$ we define $\mathcal{M}_{g, t}^{\mathcal{A}}$ to be the $A N D$ of $H_{1}^{g}$ and $H_{2}^{g}$.

Assume then that $g$ is a gate on level $l+1$ with edges from the gates $g_{1}, \ldots, g_{m}$ on level $l$ leading into $g$. According to Definition 3.4 we have in this case $S^{g}(x)=$ $\left\{v_{0}^{g}\right\} \cup \bigcup_{j=1}^{m} T^{g_{j}}(x)$ for every $x \in S$. By induction hypothesis we have already defined network architectures $\mathcal{M}_{g_{j}, t}^{\mathcal{A}}$, and hence also network architectures $\tilde{\mathcal{M}}_{g_{j}, t}^{\mathcal{A}}$ for all $t \in$ $M^{g_{j}}, j=1, \ldots, m$. For each term $t \in M^{g}$ the network architecture $\mathcal{M}_{g, t}^{\mathcal{A}}$ employs two linear threshold gates $H_{1}^{g}$ and $H_{2}^{g}$, which receive their inputs from the network architectures $\tilde{\mathcal{M}}_{g_{j}, t}^{\mathcal{A}}$ for $t \in M^{g_{j}}, j=1, \ldots, m$. The linear threshold gate $H_{1}^{g}$ has the task to check for any $x \in S$ whether $\mathcal{A}\left(v_{\mathrm{I}}^{g}\right) \leq \mathcal{A}\left(S^{g}(x), x\right)$. Obviously it can easily accomplish this task provided that for input $x$ the network architectures $\tilde{\mathcal{M}}_{g_{j}, t}^{\mathcal{A}}$ for $t \in M^{g_{j}}(j=1, \ldots, m)$ give as output the value $\mathcal{A}(t, x)$. Analogously the linear threshold gate $H_{2}^{g}$ has the task to check whether $\mathcal{A}\left(S^{g}(x), x\right)+1 \leq \mathcal{A}\left(v_{\mathrm{II}}^{g}\right)$.

If $\gamma^{g}$ outputs the constant 1 on its nontrivial piece, $\mathcal{M}_{g, v_{\text {const }}^{\mathcal{A}}}^{\mathcal{A}}$ is defined as the $A N D$ of $H_{1}^{g}$ and $H_{2}^{g}$.

If $\gamma^{g}$ computes $y \mapsto y^{k}$ on its nontrivial piece, then each $t \in M^{g}$ is of the form $v_{w_{1}, \ldots, w_{k}}^{g} \cdot \prod_{i=1}^{k} P_{i}$ for some ktuple $\left\langle w_{1} \cdot P_{1}, \ldots, w_{k} \cdot P_{k}\right\rangle \in\left(\left\{v_{0}^{g}\right\} \cup \bigcup_{j=1}^{m} M^{g_{j}}\right)^{k}$. In this case $\mathcal{M}_{g, t}^{\mathcal{A}}$ is defined as the $A N D$ of $H_{1}^{g}, H_{2}^{g}$ and of the outputs of the network architectures $\mathcal{M}_{g_{j}, w_{i} \cdot P_{i}}^{\mathcal{A}}$ for all $i \in\{1, \ldots, k\}$ and $j \in\{1, \ldots, m\}$ with $w_{i} \cdot P_{i} \in M^{g_{j}}$. 
[A word of caution: Although the variable $v_{w_{1}, \ldots, w_{k}}^{g}$ is supposed to play the role of the product of $w_{1}, \ldots, w_{k}$ and $\alpha^{g}$ (where $\alpha^{g}$ is the weight on the edge out of $g$ ), the assignment $\mathcal{A}$ will in general not satisfy $\mathcal{A}\left(v_{w_{1}, \ldots, w_{k}}^{g}\right)=\mathcal{A}\left(\alpha^{g}\right) \cdot \prod_{i=1}^{k} \mathcal{A}\left(w_{i}\right)$.]

Finally we define the network architecture $\mathcal{M}^{\mathcal{A}}$ by using as components the network architectures $\mathcal{M}_{g_{\text {out }}, t}^{\mathcal{A}}$ for all $t \in M^{g_{\text {out }}}$. The output of $\mathcal{M}^{\mathcal{A}}$ is given by a linear threshold gate $H$ that checks whether $\sum_{t: \mathcal{M}_{g_{\text {out }}, t}^{\mathcal{A}}(x)=1} \mathcal{A}(t, x) \geq \mathcal{A}\left(v^{g_{\text {out }}}\right)$.

Lemma 3.7. Assume that $S \subseteq \boldsymbol{R}^{n}$ and $\mathcal{A}$ is an arbitrary solution of $L(\mathcal{N}, S)$. Then the following holds for any gate $g$ in $\mathcal{N}$, for any term $t \in M^{g}$, and any input $x \in S$ :

(a) For network input $x$ the gate $H_{1}^{g}$ in $\mathcal{M}_{g, t}^{\mathcal{A}}$ outputs 1 if and only if $t_{1}^{g} \leq$ $W\left(S^{g}(x), x\right)$. Similarly the output of the gate $H_{2}^{g}$ in $\mathcal{M}_{g, t}^{\mathcal{A}}$ is 1 if and only if $W\left(S^{g}(x), x\right)+1 \leq t_{2}^{g}$.

(b) $t \in T^{g}(x) \Leftrightarrow\left(\mathcal{M}_{g, t}^{\overline{\mathcal{A}}}\right.$ outputs 1 for network input $\left.x\right)$.

(c) $\tilde{\mathcal{M}}_{g, t}^{\mathcal{A}}$ outputs $\mathcal{A}(t, x)$ for network input $x$.

Proof. The proof proceeds by induction on the depth of gate $g$. The claim is obvious from the definition if $g$ is of depth 1 . If $g$ is of depth $l+1>1$ we exploit the induction hypothesis for the network architectures $\mathcal{M}_{g_{j}, t}^{\mathcal{A}}$ and $\tilde{\mathcal{M}}_{g_{j}, t}^{\mathcal{A}}$ with $t \in M^{g_{j}}$ (for the immediate predecessors $g_{j}$ of gate $g$ ). Hence we may assume that gate $H_{1}^{g}$ in $\mathcal{M}_{g, t}^{\mathcal{A}}$ outputs 1 if and only if $\mathcal{A}\left(v_{\mathrm{I}}^{g}\right) \leq \mathcal{A}\left(S^{g}(x), x\right)$. Since $\mathcal{A}$ is a solution of $L(\mathcal{N}, S)$, the latter inequality holds if and only if $L(\mathcal{N}, S)$ contains the inequality $v_{\mathrm{I}}^{g} \leq\left[S^{g}(x)\right]_{x}[x]$. By Lemma 3.5 this holds if and only if $t_{1}^{g} \leq W\left(S^{g}(x), x\right)$. The claim for $H_{2}^{g}$ is verified analogously.

The least trivial case for part (b) of the claim is the case where $\gamma^{g}$ computes $y \mapsto y^{k}$ on its nontrivial piece. Then each $t \in M^{g}$ is of the form $v_{w_{1}, \ldots, w_{k}} \cdot \prod_{i=1}^{k} P_{i}$ for some $k$ tuple $\left\langle w_{1} \cdot P_{1}, \ldots, w_{k} \cdot P_{k}\right\rangle \in\left(\left\{v_{0}^{g}\right\} \cup \bigcup_{j=1}^{m} M^{g_{j}}\right)^{k}$. By definition of $T^{g}(x)$ we have $t \in T^{g}(x)$ if and only if $t_{1}^{g} \leq W\left(S^{g}(x), x\right)<t_{2}^{g}$ and $w_{i} \cdot P_{i} \in T^{g_{j}}(t)$ for all $i \in\{1, \ldots, k\}$ and $j \in\{1, \ldots, m\}$ with $w_{i} \cdot P_{i} \in M^{g_{j}}$. By construction of $\mathcal{M}_{g, t}^{\mathcal{A}}$ and by the induction hypothesis we have that $\mathcal{M}_{g, t}^{\mathcal{A}}$ outputs 1 for network input $x$ if and only if all of the preceding conditions are satisfied.

Part (c) of the claim for gate $g$ follows immediately from part (b) and the definition of $\tilde{\mathcal{M}}_{g, t}^{\mathcal{A}}$.

LEMMA 3.8. Assume that $\mathcal{N}$ is a network architecture in normal form with $n$ input variables, $S \subseteq \boldsymbol{R}^{n}$ is an arbitrary set of inputs, and $\mathcal{A}$ is an arbitrary solution of $L(\mathcal{N}, S)$. Then $\mathcal{N}$ and $\mathcal{M}^{\mathcal{A}}$ compute the same function from $S$ into $\{0,1\}$.

Proof. This is an immediate consequence of Lemmas 3.5 and 3.7. By the definition of $\mathcal{M}^{\mathcal{A}}$ the output of $\mathcal{M}^{\mathcal{A}}$ for any network input $x \in S$ is 1 if and only if

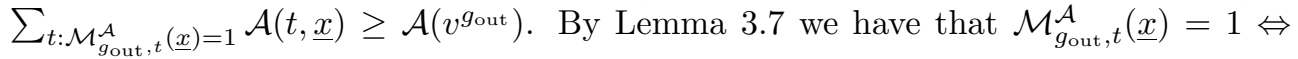
$t \in T^{\text {oout }}(x)$. Hence, since $\mathcal{A}$ is a solution of $L(\mathcal{N}, S)$, the preceding inequality holds if and only if $L(\mathcal{N}, S)$ contains the inequality $\left[\sum T^{g_{\text {out }}}(x)\right]_{x}[x] \geq v^{g_{\text {out }}}$. By definition of $L(\mathcal{N}, S)$ the latter holds if and only if $W\left(T^{g_{\text {out }}}(x), x\right) \geq W\left(v^{g_{\text {out }}}\right)$. By Lemma 3.5 the value $W\left(T^{g_{\text {out }}}(x), x\right)$ is the output of $g_{\text {out }}$ in $\mathcal{N}$ for network input $x$. Hence $W\left(T^{g_{\text {out }}}(x), x\right) \geq W\left(v^{g_{\text {out }}}\right)$ holds if and only if $\mathcal{N}$ outputs 1 for network input $x$. $\square$

We are now in a position where we can complete the proof of Theorem 3.1. Assume that a given array $\left(\mathcal{N}_{n}\right)_{n \in \mathbf{N}}$ of neural nets satisfies the assumption of Theorem 3.1 and that $\left(\alpha_{n}\right)_{n \in \mathbf{N}}$ is an arbitrary array of real-valued assignments $\alpha_{n}$ to the variable parameters in $\mathcal{N}_{n}$. One can transform the given neural nets $\left(\mathcal{N}_{n}^{\alpha_{n}}\right)_{n \in \mathbf{N}}$ into an array $\left(\hat{\mathcal{N}}_{n}^{\beta}{ }^{n}\right)_{n \in \mathbf{N}}$ of neural nets in normal form (with properties as specified above) such that 
$\hat{\mathcal{N}}_{n}^{\beta}{ }^{\beta}$ computes the same Boolean function as $\mathcal{N}_{n}^{\alpha_{n}}$. We then apply the machinery from the definition and Lemmas 3.5 to 3.8 to each neural net $\mathcal{N}:=\hat{\mathcal{N}}_{n}^{\beta}{ }^{n}$ with $S:=\{0,1\}^{n}$. By construction of $\hat{\mathcal{N}}_{n}^{\beta}{ }^{n}$ the resulting system $L\left(\mathcal{N},\{0,1\}^{n}\right)$ of inequalities has some solution over $\mathbf{R}$. We exploit here in particular that $\beta_{n}$ was chosen so that all relevant strict inequalities " $s_{1}<s_{2}$ " in computations of $\hat{\mathcal{N}}_{n}^{\beta}$ " on inputs $x \in\{0,1\}$ " were strengthened to " $s_{1}+1 \leq s_{2}$." Since $\mid \bigcup\left\{M^{g} \mid g\right.$ gate in $\left.\hat{\mathcal{N}}_{n}^{\beta}{ }^{\beta}\right\} \mid=O\left(n^{O(1)}\right)$, it follows that the number of gates in $\mathcal{M}^{\mathcal{A}}$ is bounded by $O\left(n^{O(1)}\right)$.

The number of variables in $L\left(\mathcal{N},\{0,1\}^{n}\right)$ is polynomial in $n$, and it only contains small constants. Hence by Lemma 2.4 there is a solution $\mathcal{A}$ of $L\left(\mathcal{N},\{0,1\}^{n}\right)$ that consists of rationals of the form ${ }_{t}^{s}$ (with a common integer $t$ ) such that $s$ and $t$ are integers of size $2^{O\left(n^{O(1)}\right)}$. By Lemma 3.8 the constructed network architecture $\mathcal{M}^{\mathcal{A}}$ computes the same Boolean function as $\mathcal{N}$. Furthermore all constants and parameters in $\mathcal{M}^{\mathcal{A}}$ are quotients of integers with polynomially in $n$ many bits. Thus (see $[\mathrm{SBKH}],[\mathrm{SR}]$ ) one can carry out all arithmetical operations in $\mathcal{M}^{\mathcal{A}}$ for inputs from $\{0,1\}^{n}$ by polynomial-sized digital subcircuits of constant depth with linear threshold gates (or, equivalently, with MAJORITY-gates, see [CSV]). In the resulting circuit all parameters from $\mathcal{A}$ are replaced by corresponding sequences of bits. Hence one gets in this way neural nets $\tilde{\mathcal{N}}_{n}$ which satisfy the claim of Theorem 3.1.

Remark 3.9. Sontag [S3] suggested using the quasilinearization that is achieved in the proof of Theorem 3.1 in order to also get upper bounds for the $\mathrm{VC}$ dimension over $\mathbf{R}^{n}$ by counting the number of components into which the weight space is partitioned by the hyperplanes that are defined by some arbitrary finite set $S \subseteq \mathbf{R}^{n}$ of inputs.

By letting $\alpha_{n}$ vary and keeping the neural net $\mathcal{N}_{n}$ and the input $x \in S$ fixed one gets up to $2^{O\left(n^{O(1)}\right)}$ different systems $L\left(\hat{\mathcal{N}}_{n}^{\beta}, x\right)$ in the proof of Theorem 3.1. Hence the total number $l_{n}$ of linear inequalities that arise in this way for different $x \in S$ and different parameters $\alpha_{n}$ is bounded by $|S| \cdot 2^{O\left(n^{O(1)}\right)}$. Furthermore, the total number $w_{n}$ of variables that occur in these $l_{n}$ inequalities is bounded by $O\left({ }_{n} O(1)\right)$. Therefore the hyperplanes that are associated with these $l_{n}$ inequalities partition the range $\mathbf{R}^{w_{n}}$ of the variables into at most $\sum_{k=0}^{w_{n}} \sum_{i=0}^{k}\left(\begin{array}{c}w_{n}-i \\ k-i\end{array}\right)\left(\begin{array}{c}l_{n} \\ w_{n}-i\end{array}\right)=|S|^{O\left(n^{O(1)}\right)}$ connected components (Theorem 1.3 in $[\mathrm{E}]$ ). Each $\mathcal{A} \in \mathbf{R}^{w_{n}}$ gives rise to at most $2^{O\left(n^{O(1)}\right)}$ different network architectures $\mathcal{M}^{\mathcal{A}}$ when $\mathcal{N}_{n}$ and $S$ are kept fixed, but the parameters $\alpha_{n}$ vary. Thus each $\mathcal{A} \in \mathbf{R}^{w_{n}}$ can be used to compute at most $2^{O\left(n^{O(1)}\right)}$ different functions $S \rightarrow\{0,1\}$ on the resulting circuits. Furthermore, if $\mathcal{A}$ and $\tilde{\mathcal{A}}$ belong to the same connected component of the partition of $\mathbf{R}^{w_{n}}$ then for all $\alpha_{n}$ the network architectures $\mathcal{M}^{\mathcal{A}}$ and $\mathcal{M}^{\tilde{\mathcal{A}}}$ compute the same function $S \rightarrow\{0,1\}$. Hence if $S$ is shattered by $\mathcal{N}_{n}$ (i.e., any function $S \rightarrow\{0,1\}$ can be computed by $\mathcal{N}_{n}^{\alpha_{n}}$ for suitable parameters $\left.\alpha_{n}\right)$ then $2^{|S|} \leq|S|^{O\left(n^{O(1)}\right)} \cdot 2^{O\left(n^{O(1)}\right)}$; hence $|S|=O\left(n^{O(1)}\right)$. This implies that VC dimension $\left(\mathcal{N}_{n}, \mathbf{R}^{n}\right)=O\left(n^{O(1)}\right)$.

One can apply in a similar fashion the linearization that is achieved in the proof of Theorem 2.1. Consider a neural net $\mathcal{N}$ over a graph $\langle V, E\rangle$ as in Theorem 2.1, but allow that each activation function $\gamma^{g}$ consists of $\leq p$ linear pieces with arbitrary fixed real parameters. Then one can show that VC dimension $\left(\mathcal{N}, \mathbf{R}^{n}\right)=O\left(w^{2} \log p\right)$, where $w:=|V|+|E|+1$ is the number of variable parameters in $\mathcal{N}$. It is sufficient to observe that for different $x \in S$ and different initial assignments $\alpha$ altogether at most $|S| \cdot 2^{O(w \log p)}$ linear inequalities arise in the description of the computations of the associated nets $\hat{\mathcal{N}}[c]^{\beta}$ for input $x$. The associated hyperplanes partition the "weight space" $\mathbf{R}^{w}$ for the variable parameters $\beta, c$ into $\leq|S|^{O(w)} \cdot 2^{O\left(w^{2} \log p\right)}$ connected 
components. The vectors from each connected component can be used to compute at most $2^{O(w)}$ different functions $S \rightarrow\{0,1\}$ (note that in general more than one function $S \rightarrow\{0,1\}$ can be computed because of different weights from $\{-1,0,1\}$ between computation nodes). Hence $2^{|S|} \leq|S|^{O(w)} \cdot 2^{O\left(w^{2} \log p\right)} \cdot 2^{O(w)}$ if $S$ is shattered by $\mathcal{N}$; thus $|S|=O\left(w^{2} \log p\right)$.

Subsequent to this observation from [M92] and [M93a], our polynomial upper bound for the VC dimension of analog neural nets of constant depth has been extended to neural nets of unbounded depth via an application of a well-known theorem of Milnor [GJ]. In [M93c] this result has been further generalized to yield a polynomial upper bound for the pseudodimension (see $[\mathrm{H}]$ ) of analog neural nets of arbitrary depth which takes over the role of the $\mathrm{VC}$ dimension in the case of learning on analog neural nets with real-valued outputs.

4. PAC learning on analog neural nets. We now turn to the analysis of learning on analog neural nets in Valiant's model [V] for probably approximately correct learning (PAC learning). More precisely, we consider the common extension of this model to real-valued domains due to [BEHW]. Unfortunately, most results about PAC learning on neural nets are negative (see $[\mathrm{BR}],[\mathrm{KV}]$ ). This could mean either that learning on neural nets is impossible or that the common theoretical analysis of learning on neural nets is not quite adequate.

We want to point to one somewhat problematic aspect of the traditional asymptotic analysis of PAC learning on neural nets. In analogy to the standard asymptotic analysis of the run time of algorithms in terms of the number $n$ of input bits one usually formalizes PAC learning on neural nets in exactly the same fashion. However in contrast to the common situation for computer algorithms (which typically receive their input in digital form as a long sequence of $n$ bits) for many important applications of neural nets the input is given in analog form as a vector of a small number $n$ of analog real-valued parameters. These relatively few input parameters may consist for example of sensory data, or they may be the relevant components of a longer feature vector (which were extracted by some other mechanism). If one analyzes PAC learning on neural nets in this fashion, the relevant asymptotic problem becomes a different one: can a given analog neural net with a fixed number $n$ of analog inputs approximate the target concept arbitrarily close after it has been shown sufficiently many training examples?

We show that for those types of neural nets which were considered in the preceding sections the previously discussed PAC learning problem has in fact a positive solution.

THEOREM 4.1. Let $\mathcal{N}$ be an arbitrary network architecture of order 1 as in Theorem 2.1, where the fixed parameters of the piecewise-linear activation functions may now be arbitrary reals. Let $\mathcal{C}_{\mathcal{N}}:=\left\{C \subseteq \boldsymbol{R}^{n} \mid \exists \alpha \in \boldsymbol{R}^{w} \forall x \in \boldsymbol{R}^{n}\left(\chi_{C}(x)=\mathcal{N}^{\alpha}(x)\right)\right\}$ be the associated concept class, where $\chi_{C}$ is the characteristic function of a concept C. Then $\mathcal{C}_{\mathcal{N}}$ is properly PAC learnable.

This means that one can design for the given network architecture $\mathcal{N}$ a learning algorithm $L A^{\mathcal{N}}$ such that for any distribution $Q$ over $\boldsymbol{R}^{n}$, any target concept $C_{T} \in \mathcal{C}_{\mathcal{N}}$, and any given $\varepsilon, \delta \in \boldsymbol{R}^{+}$the learning algorithm $L A^{\mathcal{N}}$ with inputs $\varepsilon$ and $\delta$ carries out in $O\left(\left(\frac{1}{\varepsilon}\right)^{O(1)},\left(\begin{array}{l}1 \\ \delta\end{array}\right)^{O(1)}\right)$ computations steps (with respect to the uniform cost criterion on a RAM) the following task: it computes a suitable number $m$ and draws some sequence $S$ of $m$ examples for $C_{T}$ according to distribution $Q$. Then it computes from $S$ an assignment $\alpha_{S} \in \boldsymbol{R}^{w}$ for the programmable parameters of $\mathcal{N}$ such that $Q\left[\left\{x \in \boldsymbol{R}^{n} \mid \chi_{C_{T}}(x) \neq \mathcal{N}^{\alpha_{S}}(x)\right\}\right] \leq \varepsilon$ with probability $\geq 1-\delta$.

Proof. We have VC dimension $\left(\mathcal{C}_{\mathcal{N}}\right)<\infty$ by Remark 3.9. Hence according 
to [BEHW], it suffices to show that for any given set $S$ of $m$ examples for $C_{T}$ one can compute from $S$ within a number of computation steps that is polynomial in $m,{ }_{\varepsilon}^{1},{ }_{\delta}^{1}$ an assignment $\alpha_{S} \in \mathbf{R}^{w}$ to the programmable parameters of $\mathcal{N}$ such that $\forall x \in S\left(\chi_{C_{T}}(x)=\mathcal{N}^{\alpha_{S}}(x)\right)$. The construction in the proof of Theorem 2.1 implies that it is sufficient if one computes instead with polynomially in $m, \frac{1}{\varepsilon}, \frac{1}{\delta}$ computation steps an assignment $\beta_{S}, c_{S}$ of parameters for the associated neural net $\hat{\mathcal{N}}$ such that $\forall x \in S\left(\chi_{C_{T}}(x)=\hat{\mathcal{N}}\left[c_{S}\right]^{\beta} S(x)\right)$. The latter task is easier because the role of the parameters $\beta, c$ in a computation of $\hat{\mathcal{N}}$ for a specific input $x$ can be described by linear inequalities (provided one knows which linear piece is used at each gate).

Nevertheless, the following technical problem remains. Although we know which output $\hat{\mathcal{N}}\left[c_{S}\right]^{\beta}$ should give for an input $x \in S$, we do not know in which way this output should be produced by $\hat{\mathcal{N}}\left[c_{S}\right]^{\beta_{S}}$. More specifically, we don't know which particular piece of each piecewise-linear activation function $\gamma^{g}$ of $\hat{\mathcal{N}}$ will be used for this computation. However, this detailed information would be needed for each $x \in S$ and for all gates $g$ of $\hat{\mathcal{N}}$ in order to describe the resulting constraints on the parameters $\beta, c$ by a system of linear inequalities.

However, one can generate a set of polynomially in $m$ many systems of linear inequalities such that at least one of these systems provides for all $x \in S$ satisfiable and sufficient constraints for $\beta, c$. By definition $\mathcal{C}_{\mathcal{N}}$ we know that there are parameters $\beta_{T}, c_{T}$ such that $\hat{\mathcal{N}}\left[c_{T}\right]^{\beta}$ computes $\chi_{C_{T}}$. Consider any inequality $I(\beta, c, x) \leq 0$ (with $I(\beta, c, x)$ linear in $\beta, c$ for fixed $x$, and linear in $x$ for fixed $\beta, c$ ) as they were introduced in the proof of Theorem 2.1 in order to describe the comparison with a threshold at some gate $g$ of $\hat{\mathcal{N}}$. The hyperplane $\left\{x \in \mathbf{R}^{n} \mid I\left(\beta_{T}, c_{T}, x\right)=0\right\}$ defines a partition of $S$ into $\left\{x \in S \mid I\left(\beta_{T}, c, x\right) \leq 0\right\}$ and $\left\{x \in S \mid I\left(\beta_{T}, c, x\right)>0\right\}$. Hence it suffices to produce (e.g., with the algorithm of [EOS]) in polynomially in $m$ many computation steps all partitions of $S$ that can be generated by as many hyperplanes as there are linear inequalities $I(\beta, c, x) \leq 0$ in the proof of Theorem 2.1. One of these partitions will agree with the partition of $S$ that is defined by the hyperplanes $\left\{x \in \mathbf{R}^{n} \mid I\left(\beta_{T}, c_{T}, x\right)=0\right\}$ for the "correct values" $\beta_{T}, c_{T}$ of the parameters. Each of these partitions corresponds to a guess which linear pieces of the activation functions $\gamma^{g}$ of $\hat{\mathcal{N}}$ are used for the different inputs $x \in S$, and hence it defines a unique system of linear inequalities in $\beta, c$ (with the inputs $x \in S$ as fixed coefficients). Furthermore, it is guaranteed that one of these guesses is correct for $\beta_{T}, c_{T}$.

For each of the resulting polynomially in $m$ many systems of inequalities we apply the method of the proof of Lemma 2.4 (i.e., we reduce the solution of each system of inequalities to the solution of polynomially in $m$ many systems of linear equalities), or we apply Megiddo's polynomial time algorithm for linear programming in a fixed dimension $[\mathrm{Me}]$ in order to find values $\beta_{s}, c_{s}$ for which $\hat{\mathcal{N}}\left[c_{s}\right]^{\beta_{s}}$ gives the desired outputs for all $x \in S$. By construction, this algorithm will succeed for at least one of the selected system of inequalities.

Remark 4.2. Assume $\mathcal{N}$ is some arbitrary network architecture of order 1 according to Definition 1.1 with arbitrary piecewise-linear activation functions, and $\mathcal{N}$ does not satisfy the condition that all computation nodes of $\mathcal{N}$ have fan-out $\leq 1$. Then Theorem 4.1 does not show that $C_{\mathcal{N}}$ is properly PAC learnable. However, it implies that $C_{\mathcal{N}}$ is PAC learnable, with $\mathcal{C}_{\mathcal{N}^{\prime}}$ for a somewhat larger network architecture $\mathcal{N}^{\prime}$ of the same depth used as hypothesis class (see Remark 2.2 for the definition of $\mathcal{N}^{\prime}$ ).

Note that this result may lead toward a theoretical explanation of an effect that has been observed in many experiments: one often achieves better learning results on artificial neural nets if one uses a neural net with somewhat more units than necessary 
(i.e., necessary in order to compute the target concept on the neural net).

THEOREM 4.3. Let $\mathcal{N}$ be an arbitrary network architecture with arbitrary piecewisepolynomial activation functions and arbitrary polynomial gate inputs $Q^{g}\left(y_{1}, \ldots, y_{m}\right)$. Then the associated concept class $\mathcal{C}_{\mathcal{N}}$ is PAC learnable with a hypothesis class of the form $\mathcal{C}_{\tilde{\mathcal{N}}}$ for a somewhat larger network architecture $\tilde{\mathcal{N}}$.

Proof. One can reduce this problem to the case of network architectures with linear gate inputs as indicated at the beginning of the proof of Theorem 3.1. One uses as hypotheses, sets which are defined by a network architecture $\tilde{\mathcal{N}}$ of the same structure as the network architecture $\mathcal{M}^{\mathcal{A}}$ in the proof of Theorem 3.1. For this network architecture $\tilde{\mathcal{N}}$ one can express the constraints on the assignment $\mathcal{A}$ by linear inequalities. Remark 3.9 implies that $\mathrm{VC}$ dimension $\left(\tilde{\mathcal{N}}, \mathbf{R}^{n}\right)<\infty$.

One applies the method from the proof of Lemma 2.4 in a manner analogous to the proof of Theorem 4.1 or linear programming in a fixed dimension [Me] to polynomially in $m$ many systems of linear inequalities. There is one small obstacle in generating the associated partitions of $S$ since the corresponding inequalities are not linear in the circuit inputs $x$. One overcomes this difficulty by going to an input space of higher dimension (where the variables represent monomials of bounded degree in the original variables).

Remark 4.4. It is shown in [M93c] that the positive learning results of this section can be extended to analog neural nets with real-valued outputs. Furthermore it is shown in that paper that these learning results can be extended to Haussler's refinement $[\mathrm{H}]$ of Valiant's model $[\mathrm{V}]$, where no a priori assumptions about the target function are required and where arbitrary noise in the training examples is permitted.

Acknowledgments. We would like to thank Eduardo D. Sontag for drawing our attention to the problem of finding upper bounds for neural nets with $\pi$-gates and for his insightful comments. We thank Peter Auer, Franz Aurenhammer, Philip M. Long, and Gerhard Wöginger for various helpful discussions on this research.

\section{REFERENCES}

[A] Y. S. Abu-Mostafa, The Vapnik-Chervonenkis dimension: Information versus complexity in learning, Neural Comput., 1 (1989), pp. 312-317.

[B] P. L. BARTLETT, Lower bounds on the Vapnik-Chervonenkis dimension of multi-layer threshold networks, in Proc. 5th Annual ACM Conference on Computational Learning Theory, ACM, New York, 1993, pp. 144-150.

[BH] E. B. BAum AND D. HAussler, What size net gives valid generalization?, Neural Comput., 1 (1989), pp. 151-160.

[BR] A. Blum AND R. L. Rivest, Training a 3-node neural network is NP-complete, in Proc. 1988 Workshop on Computational Learning Theory, Morgan Kaufmann, San Mateo, CA, 1988, pp. 9-18.

[BEHW] A. Blumer, A. Ehrenfeucht, D. Haussler, And M. K. Warmuth, Learnability and the Vapnik-Chervonenkis dimension, J. Assoc. Comput. Mach., 36 (1989), pp. 929965.

[CSV] A. K. Chandra, L. Stockmeyer, and U. Vishkin, Constant depth reducibility, SiAM J. Comput., 13 (1984), pp. 423-439.

[DS] B. DasGupta And G. Schnitger, The power of approximating: A comparison of activation functions, in Advances in Neural Information Processing Systems, Vol. 5, Morgan Kaufmann, San Mateo, 1993, pp. 615-622.

[DR] R. Durbin And D. E. Rumelhart, Product units: A computationally powerful and biologically plausible extension to backpropagation networks, Neural Computation, 1 (1989), pp. 133-142.

[E] H. Edelsbrunner, Algorithms in Combinatorial Geometry, Springer-Verlag, Berlin, 1987. 
[EOS] H. Edelsbrunner, J. O'Rourke, And R. Seidel, Constructing arrangements of lines and hyperplanes with applications, SIAM J. Comput., 15 (1986), pp. 341-363.

[GJ] P. Goldberg And M. Jerrum, Bounding the Vapnik-Chervonenkis dimension of concept classes parameterized by real numbers, in Proc. 5th Annual ACM Conference on Computational Learning Theory, ACM, New York, 1993, pp. 361-369.

[GHR] M. Goldmann, J. HÅstAD, AND A. RAZBOROv, Majority gates vs. general weighted threshold gates, in Proc. 7th Structure in Complexity Theory Conference, IEEE Computer Society Press, Los Alamitos, CA, 1992, pp. 2-13.

[HMPST] A. Hajnal, W. Maass, P. Pudlák, M. Szegedy, and G. Turan, Threshold circuits of bounded depth, J. Comput. System Sci., 46 (1993), pp. 129-154.

[Has] J. HÅSTAD, On the size of weights for threshold gates, SIAM J. Discrete Math., 7 (1994), pp. $484-492$.

[H] D. HAussler, Decision theoretic generalizations of the PAC model for neural nets and other learning applications, Inform. and Comput., 100 (1992), pp. 78-150.

[Ho] J. J. Hopfield, Neurons with graded response have collective computational properties like those of two-state neurons, Proc. Nat. Acad. Sci. U.S.A., (1984), pp. 3088-3092.

[J] D. S. Johnson, A catalog of complexity classes, in Handbook of Theoretical Computer Science, A, J. van Leeuwen, ed., MIT Press, Cambridge, MA, 1990, pp. 67-161.

[KV] M. Kearns and L. Valiant, Cryptographic limitations on learning boolean formulae and finite automata, in Proc. 21st ACM Symposium on Theory of Computing, ACM, New York, 1989, pp. 433-444.

[K93] P. Kolran, A weak version of the Blum, Shub, Smale model, in Proc. 34th Annual Symposium on Foundations of Computer Science, IEEE Computer Society Press, Los Alamitos, CA, 1993, pp. 486-495.

[K94] P. KoIRAn, Efficient learning of continuous neural networks, Proc. 7th Annual ACM Conference on Computational Learning Theory, 1994, ACM, New York, 1994, pp. 348-355.

[L] R. P. LIPPMANN, An introduction to computing with neural nets, IEEE ASSP Magazine, 1987, pp. 4-22.

[M92] W. MAAss, Bounds for the computational power and learning complexity of analog neural nets, IIG Report 349, Technische Universität Graz, Graz, Austria, 1992.

[M93a] W. MAAss, Bounds for the computational power and learning complexity of analog neural nets (extended abstract), in Proc. 25th ACM Symposium on the Theory of Computing, 1993, pp. 335-344.

[M93b] W. MAAss, Neural nets with superlinear VC-dimension, in Proc. International Conference on Artificial Neural Networks, Springer-Verlag, Berlin, 1994, pp. 581-584. Neural Comput., 6 (1994), pp. 877-884.

[M93c] W. MAAss, Agnostic PAC-learning of functions on analog neural nets, Neural Comput., 7 (1995), pp. 902-926.

[MSS] W. MaAss, G. Schnitger, and E. D. Sontag, On the computational power of sigmoid versus boolean threshold circuits, in Proc. 32nd Annual IEEE Symposium on Foundations of Computer Science, IEEE Computer Society Press, Los Alamitos, CA, 1991, pp. $767-776$.

[MT] W. MaAss And G. Turan, How fast can a threshold gate learn?, in Computational Learning Theory and Natural Learning Systems: Constraints and Prospects, G. Drastal, S. J. Hanson, and R. Rivest, eds., MIT Press, Cambridge, MA, 1994, pp. 381-414.

[MR] J. L. McClelland and D. E. Rumelhart, Parallel Distributed Processing, Vol. 2, MIT Press, Cambridge, MA, 1986.

[Me] N. Megiddo, Linear programming in linear time when the dimension is fixed, J. Assoc. Comput. Mach., 31 (1984), pp. 114-127.

[MP] M. Minsky And S. PAPERT, Perceptrons: An Introduction to Computational Geometry, expanded Ed., MIT Press, Cambridge, MA, 1988.

[MD] J. MoOdy AND C. J. DARKEN, Fast learning in networks of locally tuned processing units, Neural Computation, 1 (1989), pp. 281-294.

[Mu $\quad$ S. Muroga, Threshold Logic and Its Applications, John Wiley, New York, 1971.

[Ni] N. J. Nilsson, Learning Machines, McGraw-Hill, New York, 1971.

[PS] I. Parberry and G. Schnitger, Parallel computation with threshold functions, in Lecture Notes in Computer Science, Vol. 223, Springer-Verlag, Berlin, 1986, pp. 272290.

[PG] T. Poggio And F. Girosi, Networks for approximation and learning, Proc. IEEE, 78 (1990), pp. 1481-1497. 
[R] F. Rosenblatt, Principles of Neurodynamics, Spartan Books, New York, 1988.

[RM] D. E. Rumelhart and J. L. McClelland, Parallel Distributed Processing, Vol. 1, MIT Press, Cambridge, MA, 1986.

[Sch] A. SchriJver, Theory of Linear and Integer Programming, John Wiley, New York, 1986.

[SS] H. T. Spiegelmann and E. D. Sontag, Neural Networks with Real Weights: Analog Computational Complexity, Report SYCON-92-05, Rutgers Center for Systems and Control, Rutgers University, New Brunswick, NJ, 1992.

[SBKH] K. Y. Siu, J. Bruck, T. Kailath, And T. Hofmeister, Depth efficient neural networks for division and related problems, IEEE Trans. Inform. Theory, 39 (1993), pp. 946956.

[SR] K. Y. Siu And V. Roychowdhury, On Optimal Depth Threshold Circuits for Multiplication and Related Problems, Technical Report ECE-92-05, University of California at Irvine, Irvine, CA, 1992.

[S1] E. D. SontAG, Remarks on interpolation and recognition using neural nets, in Advances in Neural Information Processing Systems 3, R. P. Lippmann, J. Moody, D. S. Touretzky, eds., Morgan Kaufmann, San Mateo, CA, 1991, pp. 939-945.

[S2] E. D. Sontag, Feedforward nets for interpolation and classification, J. Comput. System Sci., 45 (1992), pp. 20-48.

[S3] E. D. SonTAG, private communication, July, 1992.

[V] L. G. Valiant, A theory of the learnable, Comm. Assoc. Comput. Mach., 27 (1984), pp. 1134-1142. 
Copyright $\odot 2003$ EBSCO Publishing 